\title{
An Investigation into the One-Pot Heck Olefination-Hydrogenation Reaction
}

\author{
Kimberly Geoghegan, Susan Kelleher and Paul Evans* \\ Centre for Synthesis and Chemical Biology, School of Chemistry and Chemical Biology, \\ University College Dublin, Dublin 4, Ireland.E-mail: paul.evans@ucd.ie
}

\section{Contents:}

Scanned Spectra

S2-32 


\section{Proton and carbon NMR spectra:}

1-Methyl-8-thia-9-aza-tricyclo[7.2.1.0 $0^{2,7}$ dodeca-2(7),3,5-triene 8,8-dioxide 11c (400 MHz, $\left.\mathrm{CDCl}_{3}\right)$

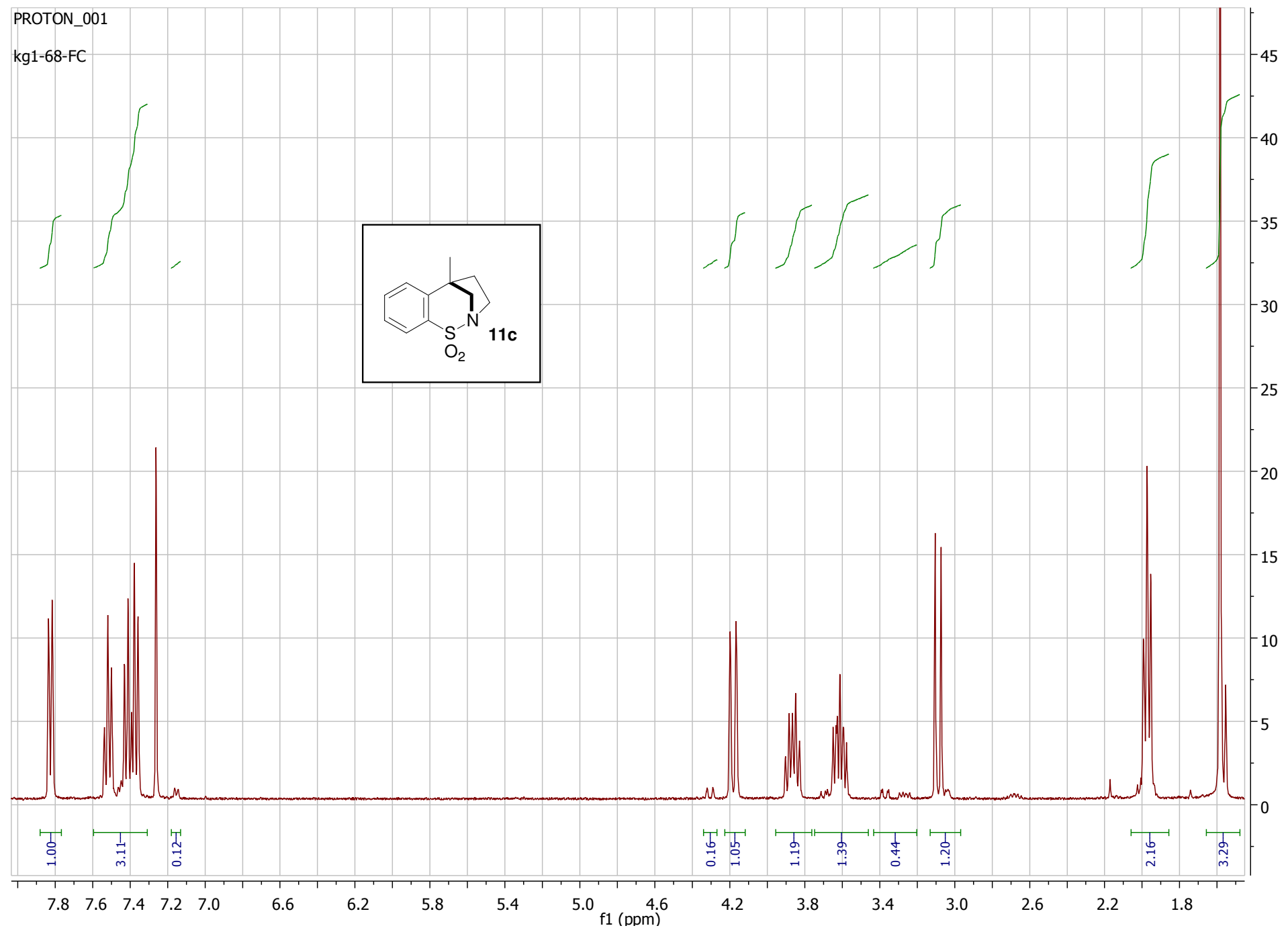


1-Methyl-8-thia-9-aza-tricyclo[7.2.1.0 $0^{2,7}$ dodeca-2(7),3,5-triene 8,8-dioxide 11c $\left(100 \mathrm{MHz}, \mathrm{CDCl}_{3}\right)$

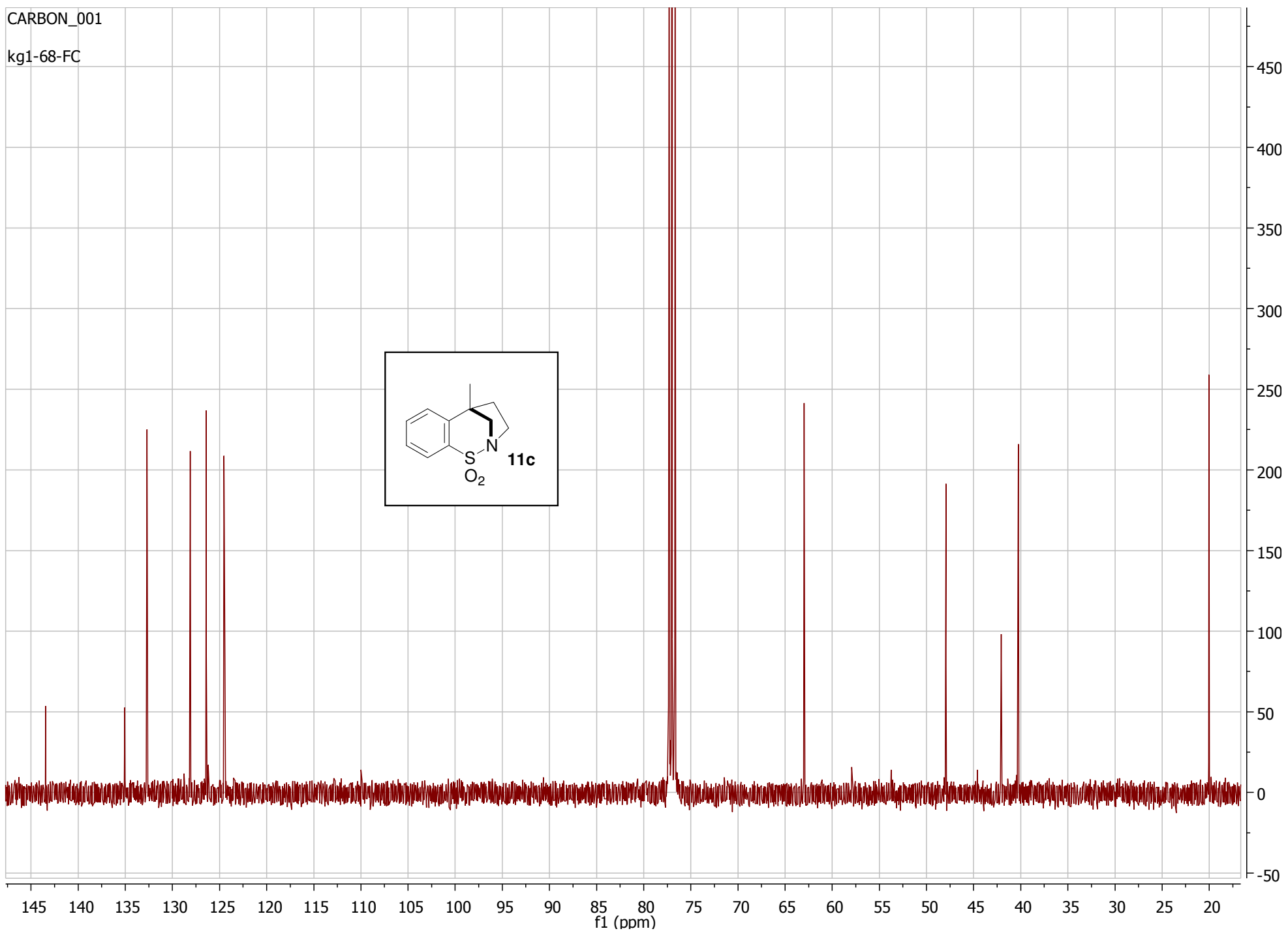


1-(2-Bromo-4,5-dimethoxybenzenesulfonyl)-3-methyl-2,5-dihydro-1 $H$-pyrrole 9d $\left(400 \mathrm{MHz}, \mathrm{CDCl}_{3}\right)$

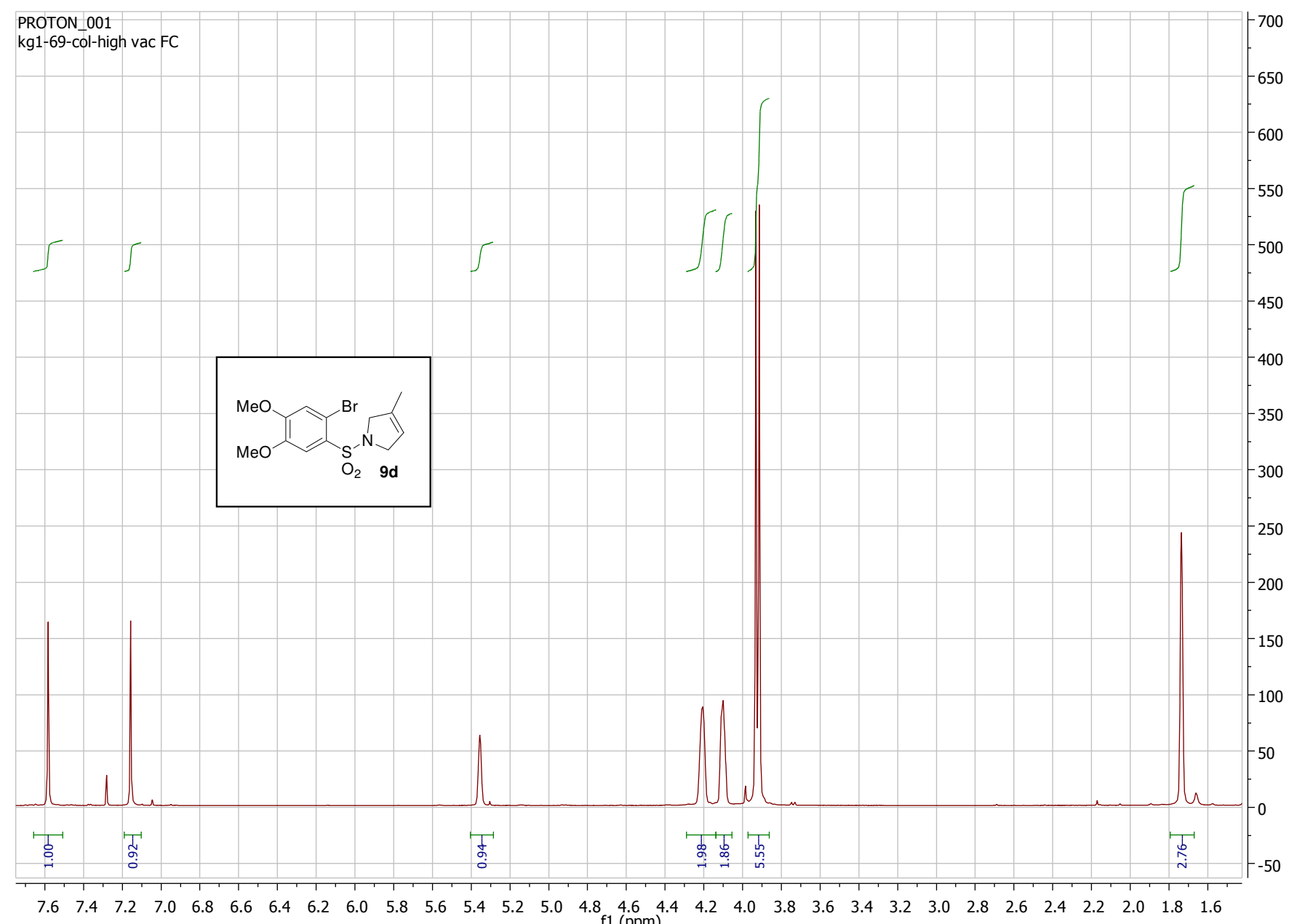


1-(2-Bromo-4,5-dimethoxybenzenesulfonyl)-3-methyl-2,5-dihydro-1 $H$-pyrrole 9d $\left(100 \mathrm{MHz}, \mathrm{CDCl}_{3}\right)$

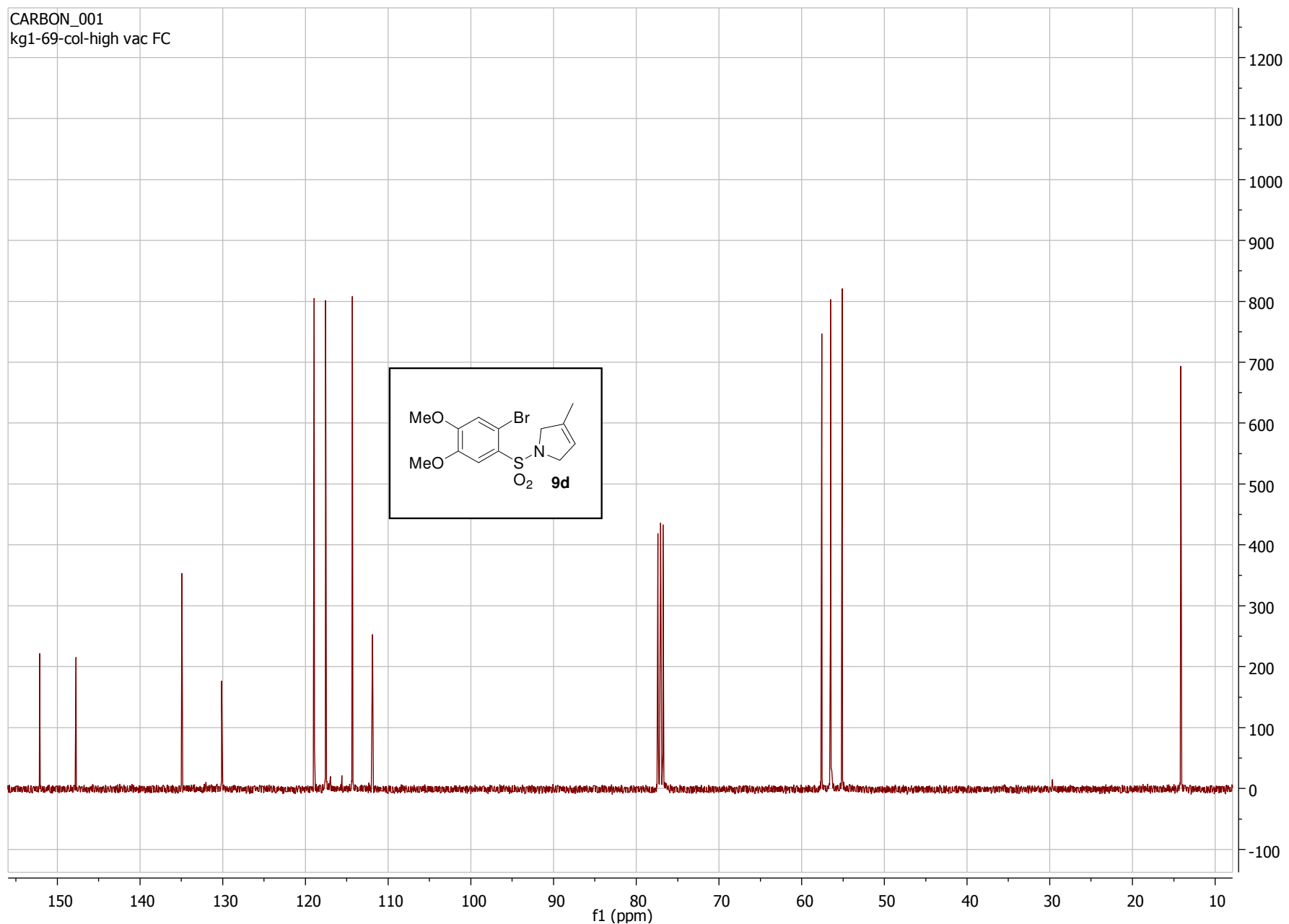


4,5-Dimethoxy-1-methyl-8-thia-9-aza-tricyclo[7.2.1.0 $\left.{ }^{2,7}\right]$ dodeca-2(7),3,5,10-tetraene 8,8-dioxide 10d (400 $\left.\mathrm{MHz} \mathrm{CDCl}_{3}\right)$

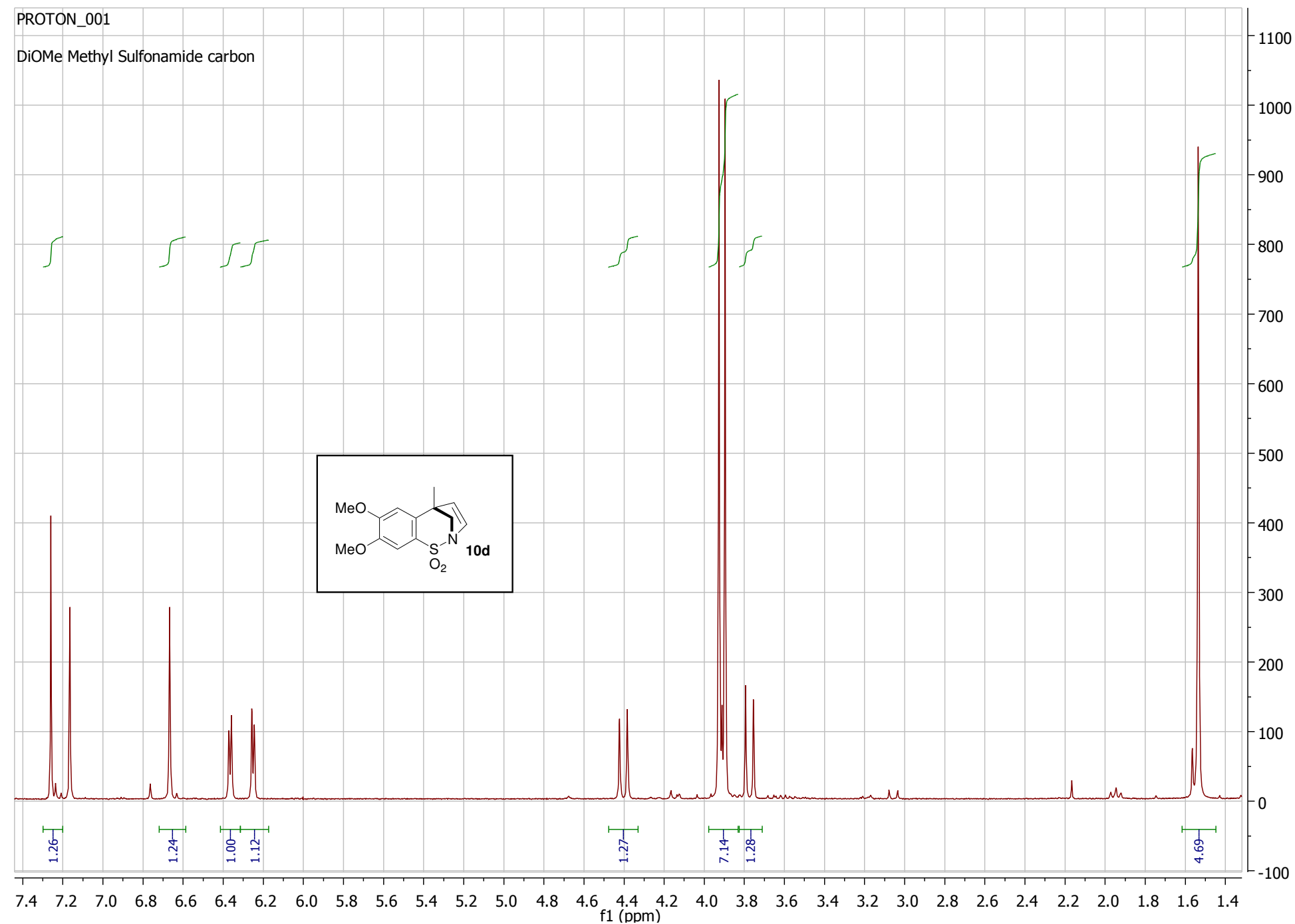


4,5-Dimethoxy-1-methyl-8-thia-9-aza-tricyclo[7.2.1.0 $\left.0^{2,7}\right]$ dodeca-2(7),3,5,10-tetraene 8,8-dioxide $\mathbf{1 0 d}\left(100 \mathrm{MHz}^{\mathrm{CDCl}} \mathrm{CD}_{3}\right)$

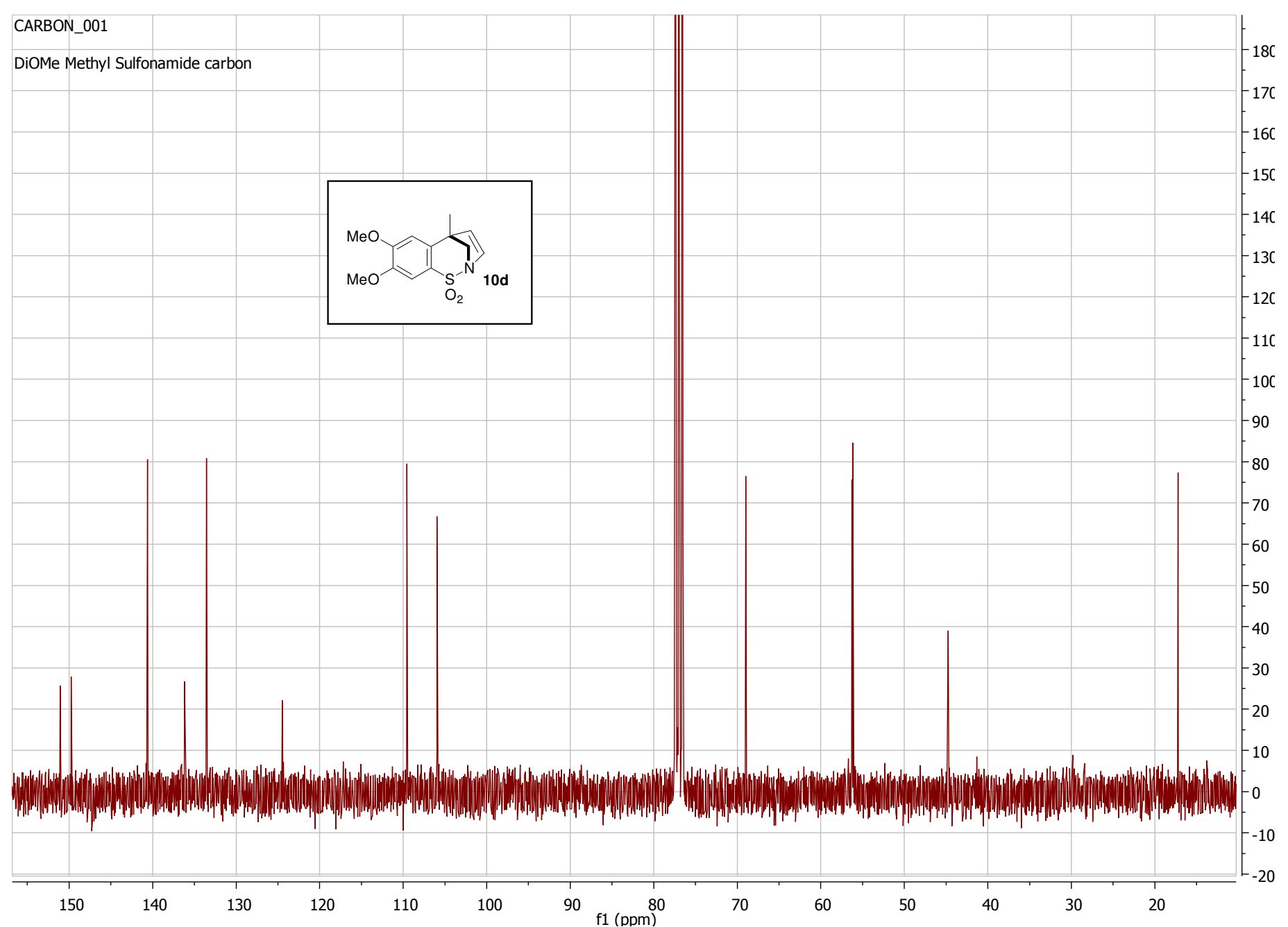


4,5-Dimethoxy-1-methyl-8-thia-9-aza-tricyclo[7.2.1.0 $\left.0^{2,7}\right]$ dodeca-2(7),3,5-triene 8,8-dioxide $11 d\left(400 \mathrm{MHz}, \mathrm{CDCl}_{3}\right)$

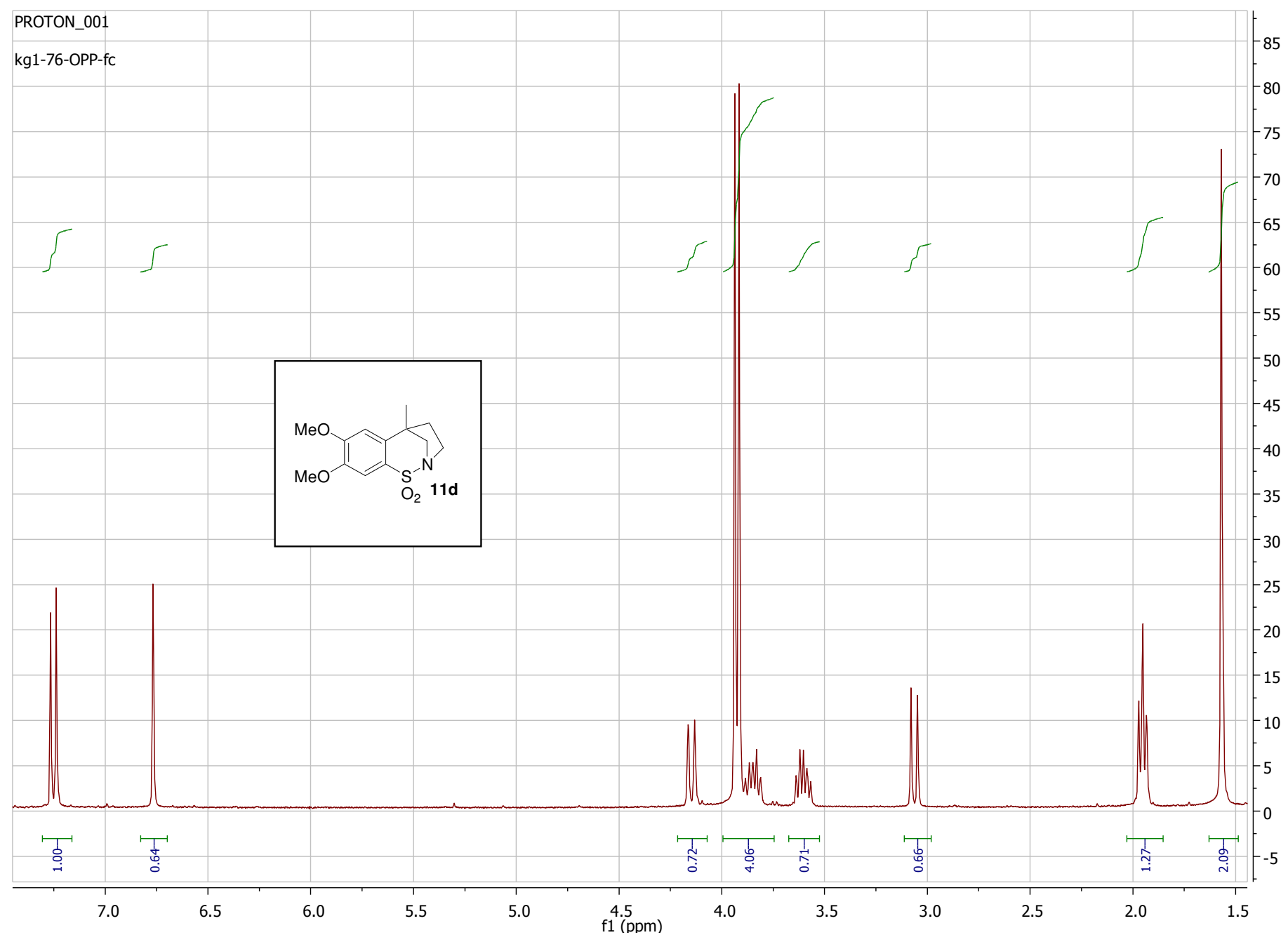


4,5-Dimethoxy-1-methyl-8-thia-9-aza-tricyclo[7.2.1.0 $\left.0^{2,7}\right]$ dodeca-2(7),3,5-triene 8,8-dioxide $11 d\left(100 \mathrm{MHz}, \mathrm{CDCl}_{3}\right)$

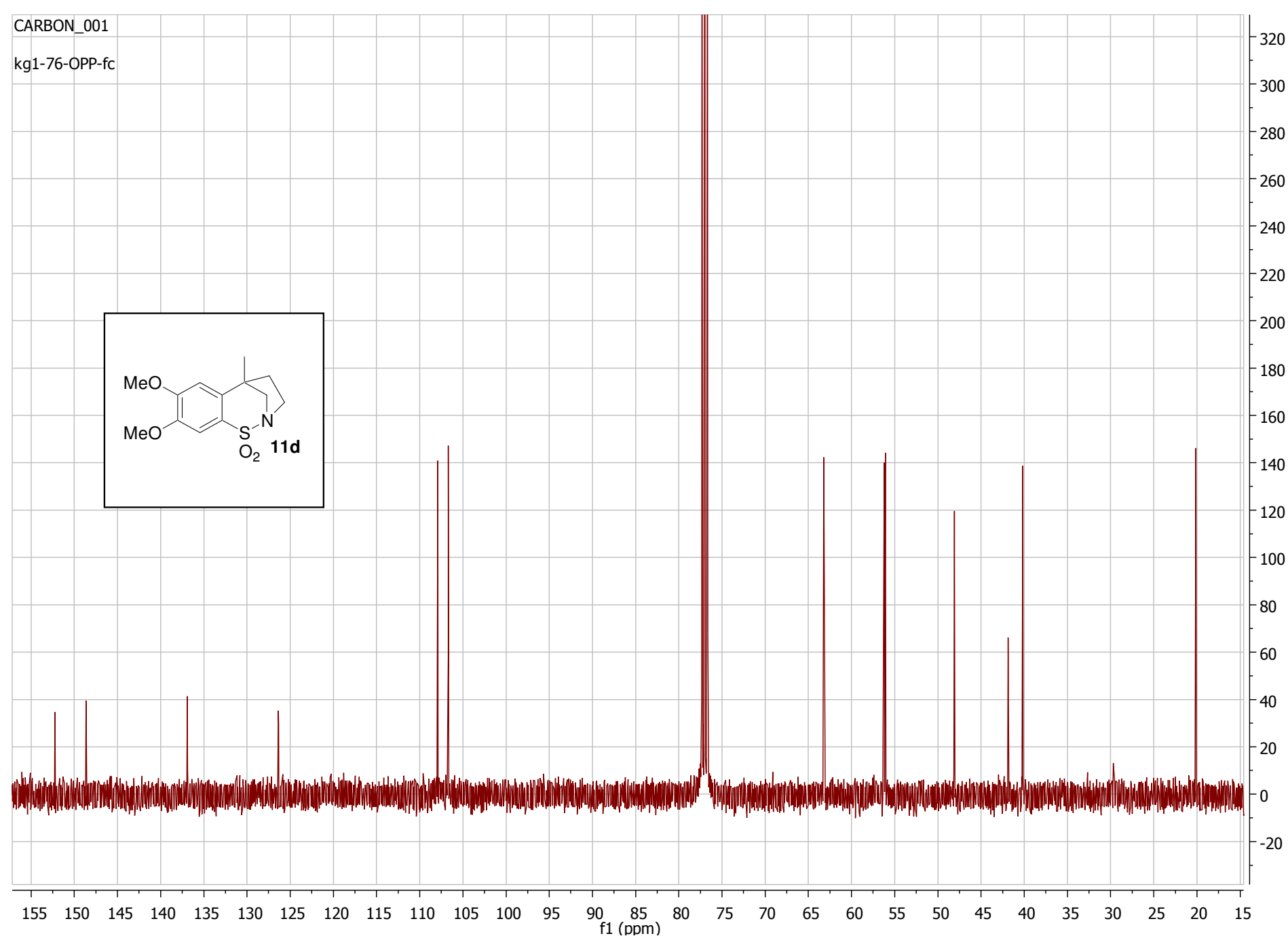


1-(2-Bromobenzenesulfonyl)-2,4,5,6,7,7a-hexahydro- $1 H$-indole $9 e\left(400 \mathrm{MHz}, \mathrm{CDCl}_{3}\right)$

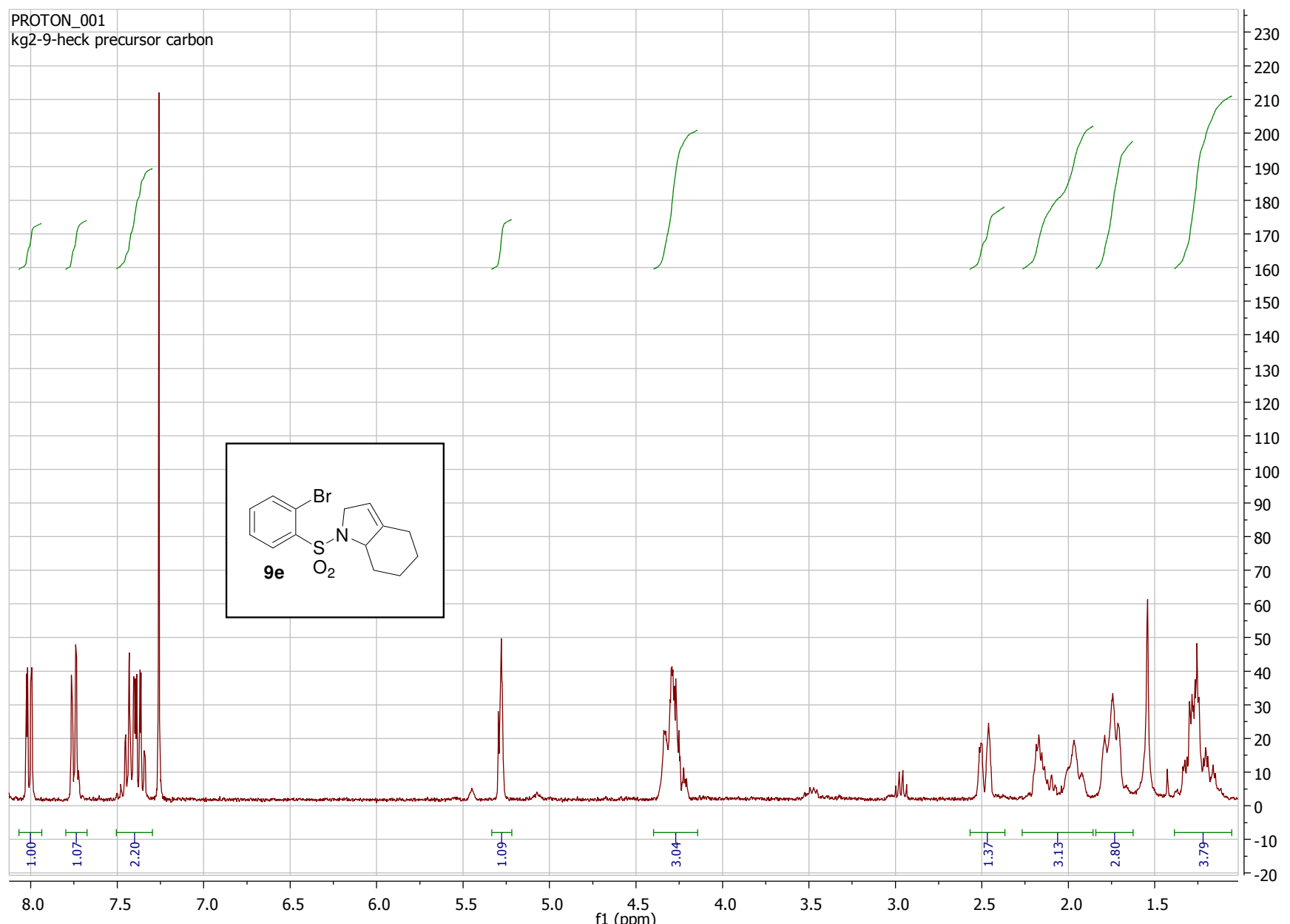


1-(2-Bromobenzenesulfonyl)-2,4,5,6,7,7a-hexahydro- $1 H$-indole $9 e\left(100 \mathrm{MHz}, \mathrm{CDCl}_{3}\right)$

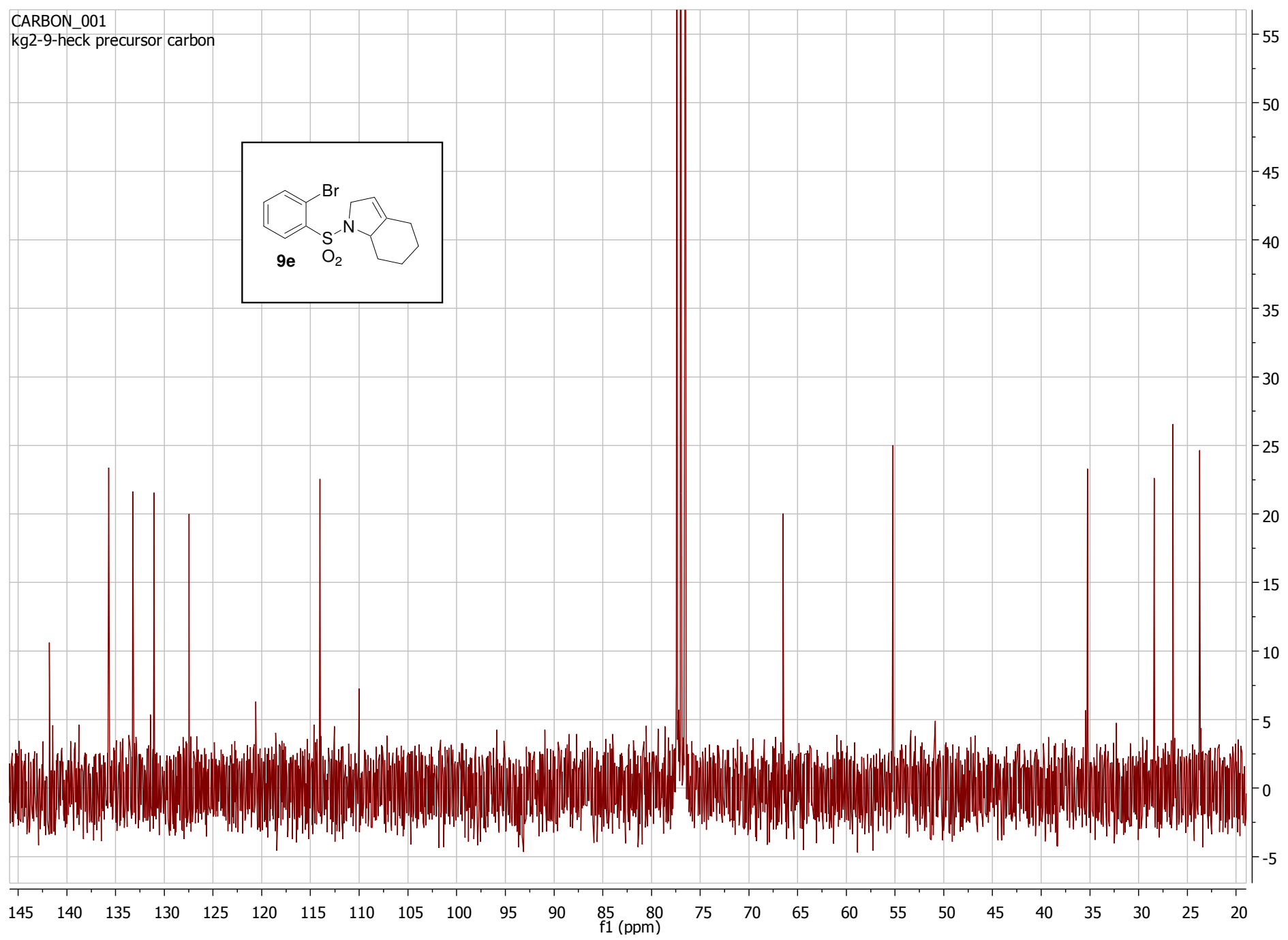


( \pm )-(4a $R, 10 \mathrm{a} S)-4 \mathrm{a}, 10$-etheno-2,3,4,4a,10,10a-hexahydro-1H-9-thia-10-azaphenanthrene 9,9-dioxide $\mathbf{1 0 e}\left(400 \mathrm{MHz}, \mathrm{CDCl}_{3}\right)$

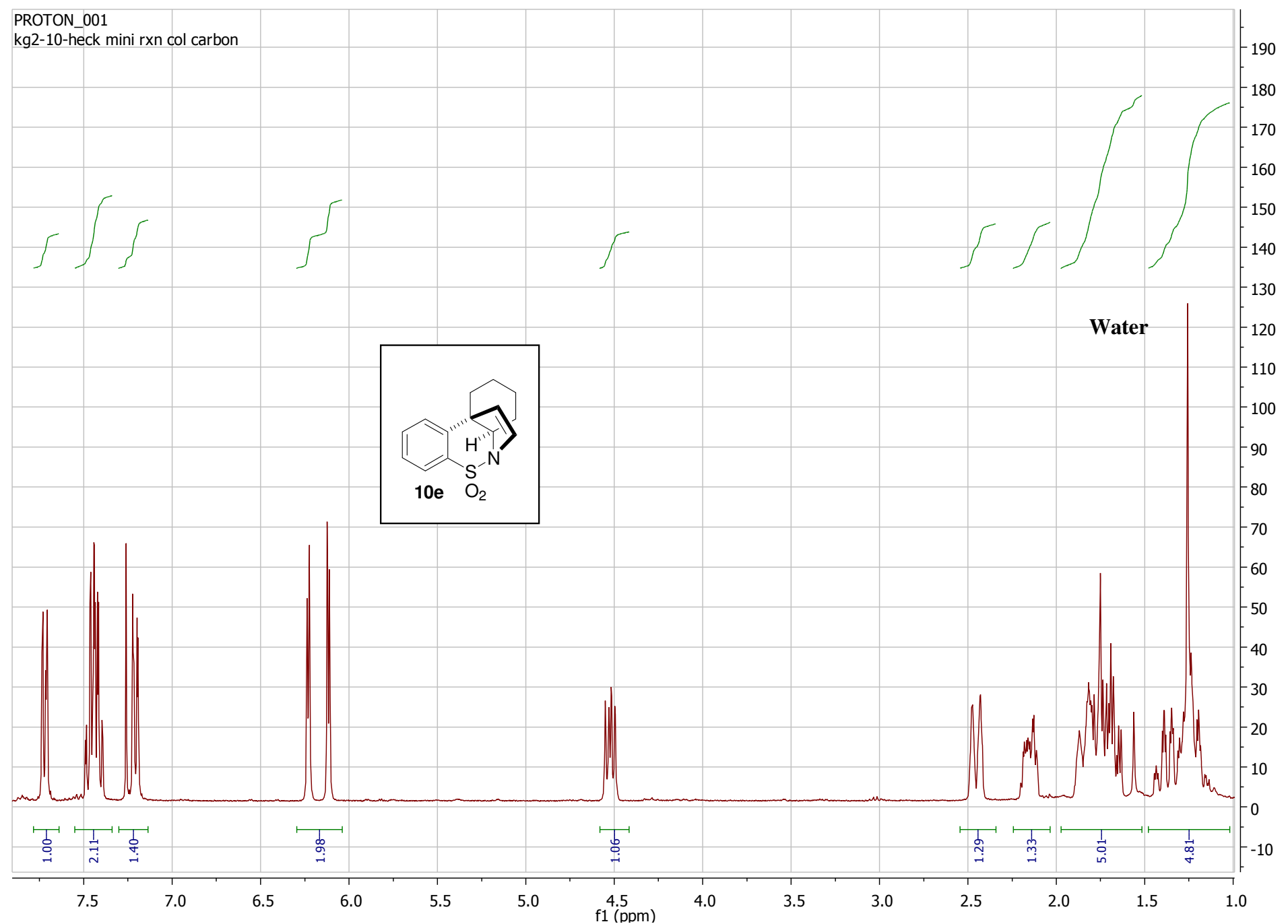


( \pm )-(4a $R, 10 \mathrm{a} S)-4 \mathrm{a}, 10$-etheno-2,3,4,4a,10,10a-hexahydro-1H-9-thia-10-azaphenanthrene 9,9-dioxide $10 \mathrm{e}\left(100 \mathrm{MHz} \mathrm{CDCl}_{3}\right)$

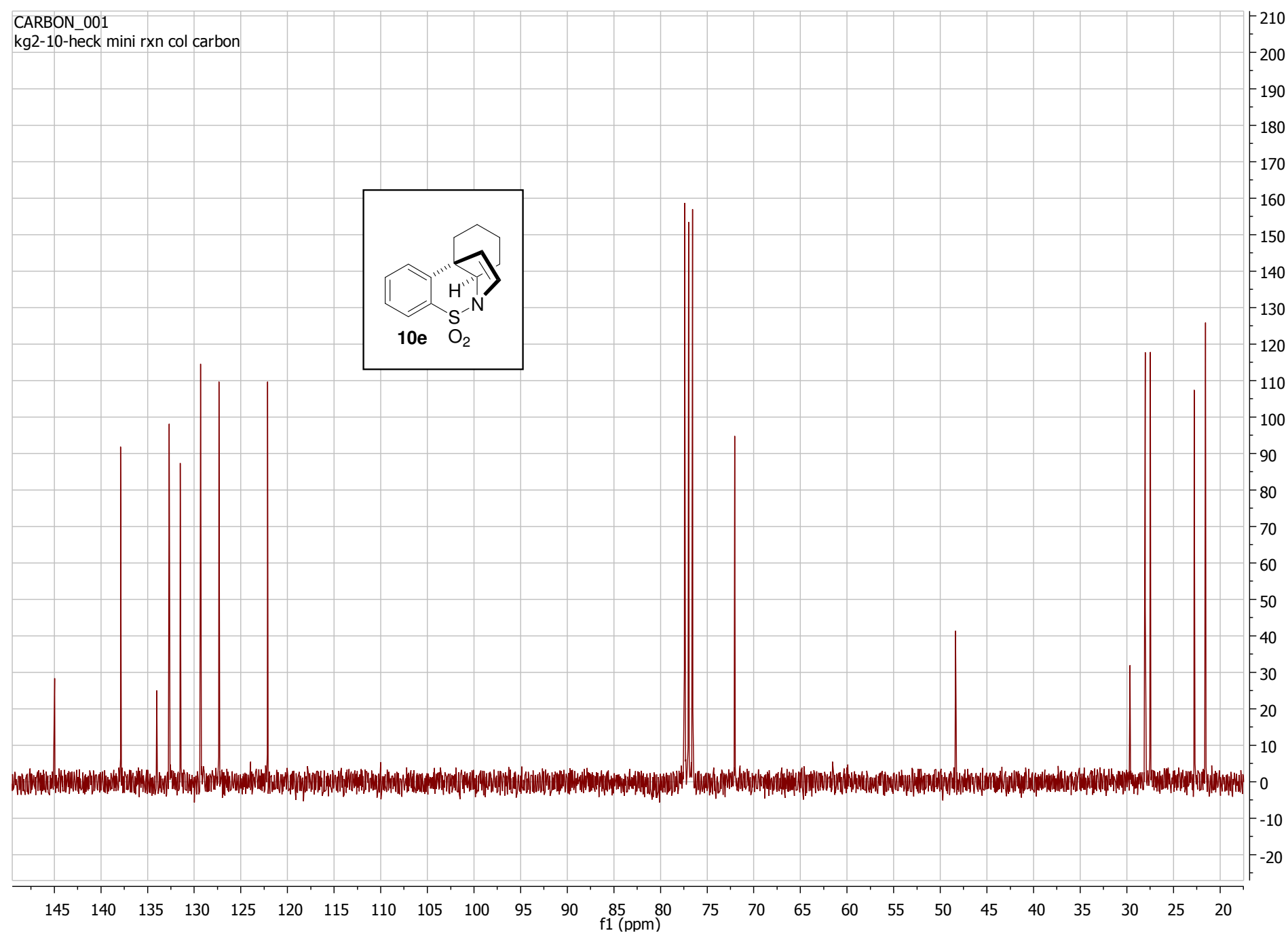




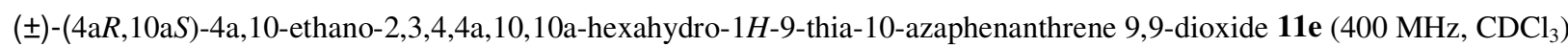

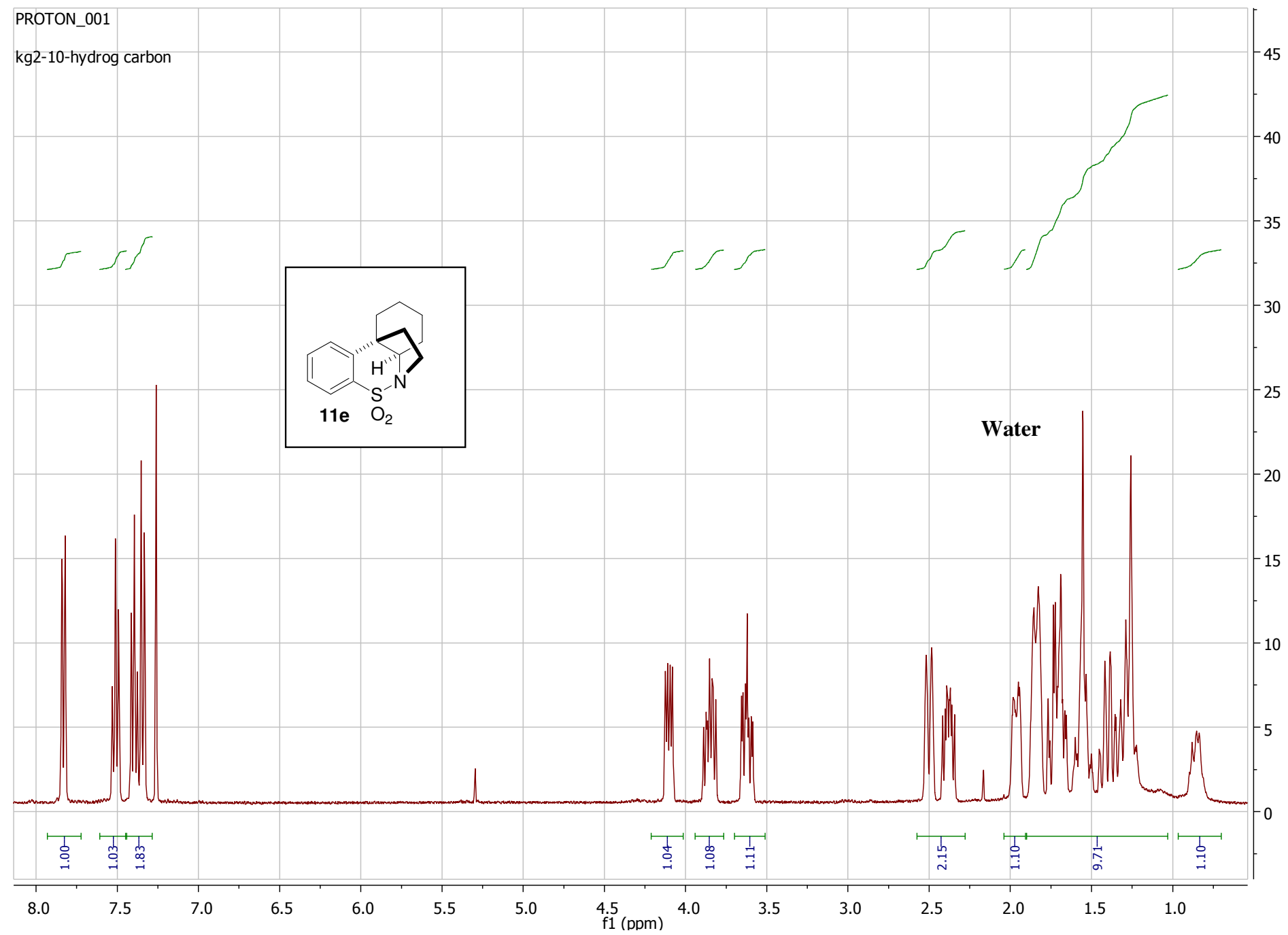




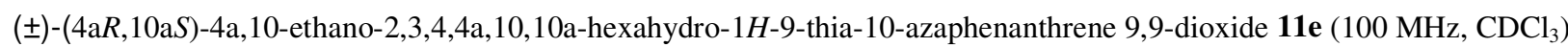

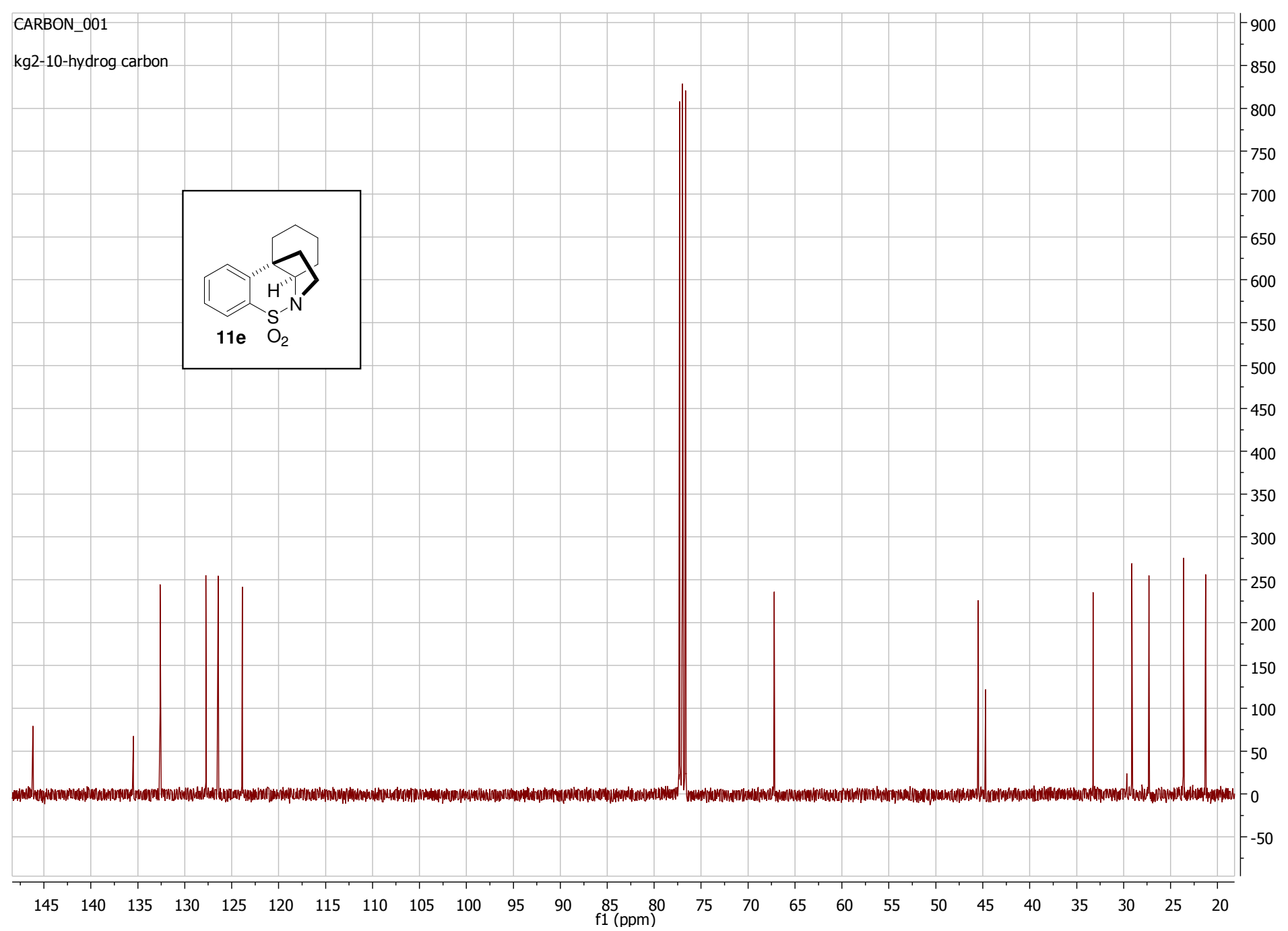




\section{2-Phenyltetrahydrofuran $\mathbf{1 5 h}\left(400 \mathrm{MHz}, \mathrm{CDCl}_{3}\right)$}

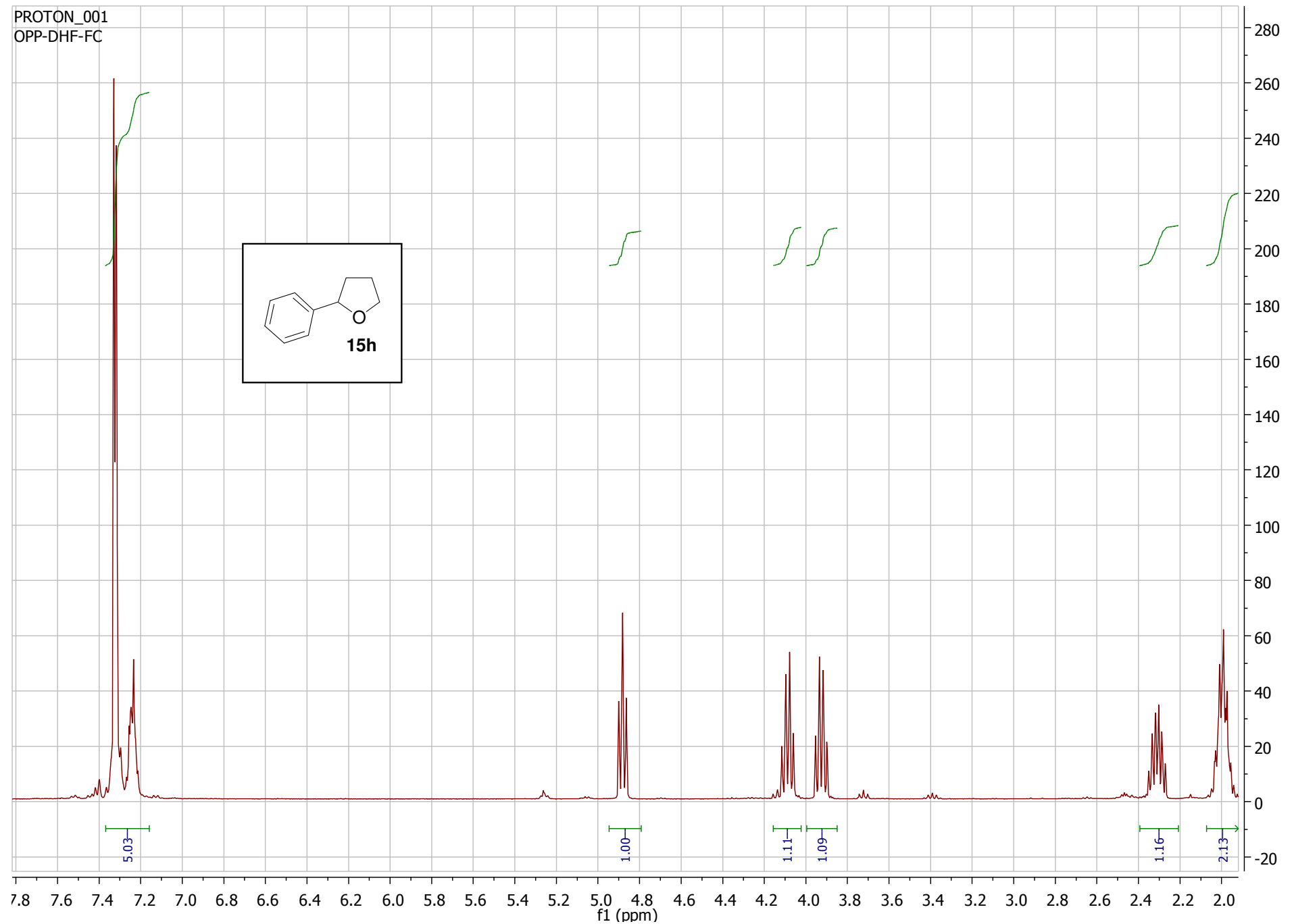


2-Phenyltetrahydrofuran $\mathbf{1 5 h}\left(100 \mathrm{MHz}, \mathrm{CDCl}_{3}\right)$

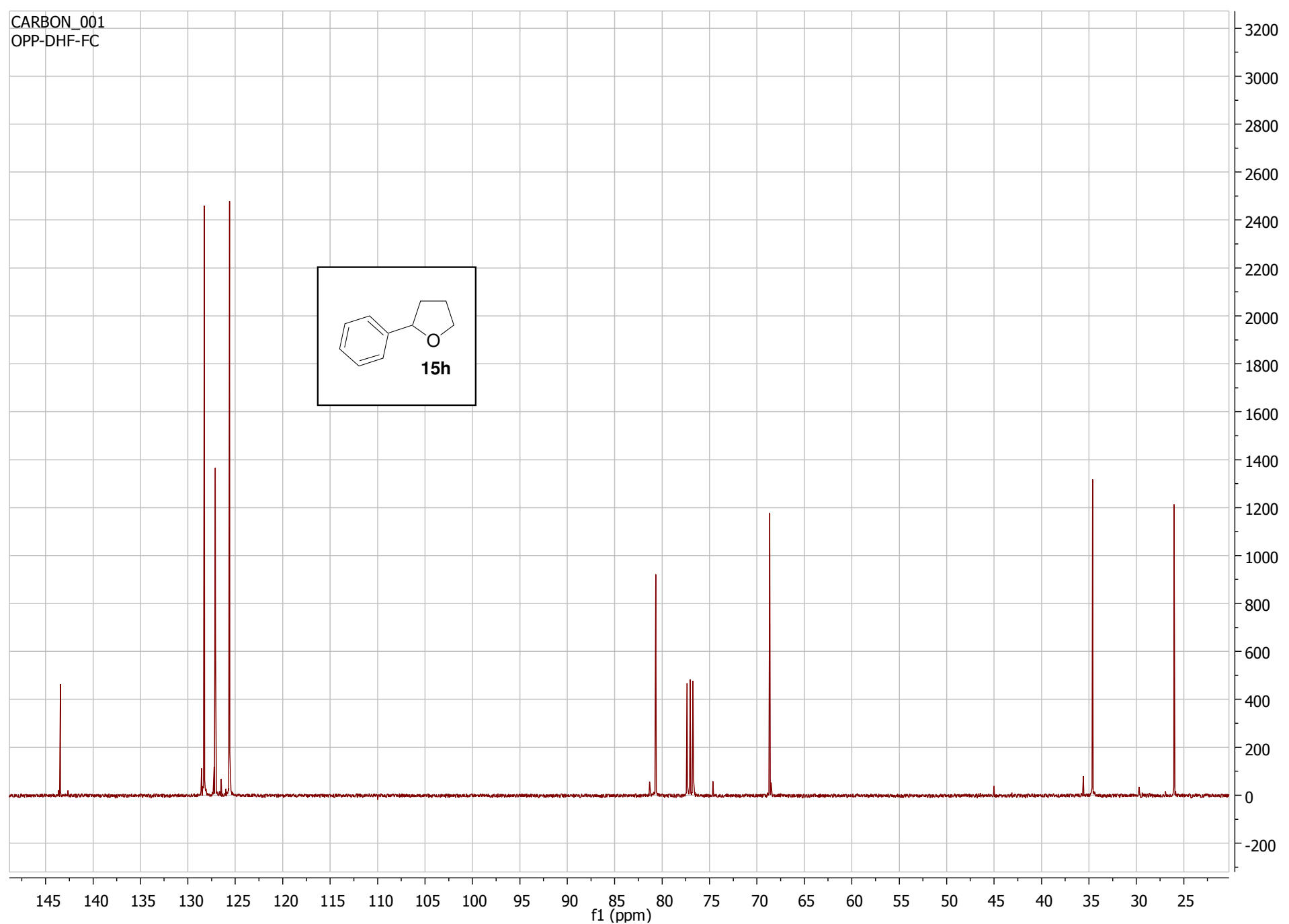


Methyl 3-(1-naphthyl)propanoate 17a (400 MHz, $\mathrm{CDCl}_{3}$ )

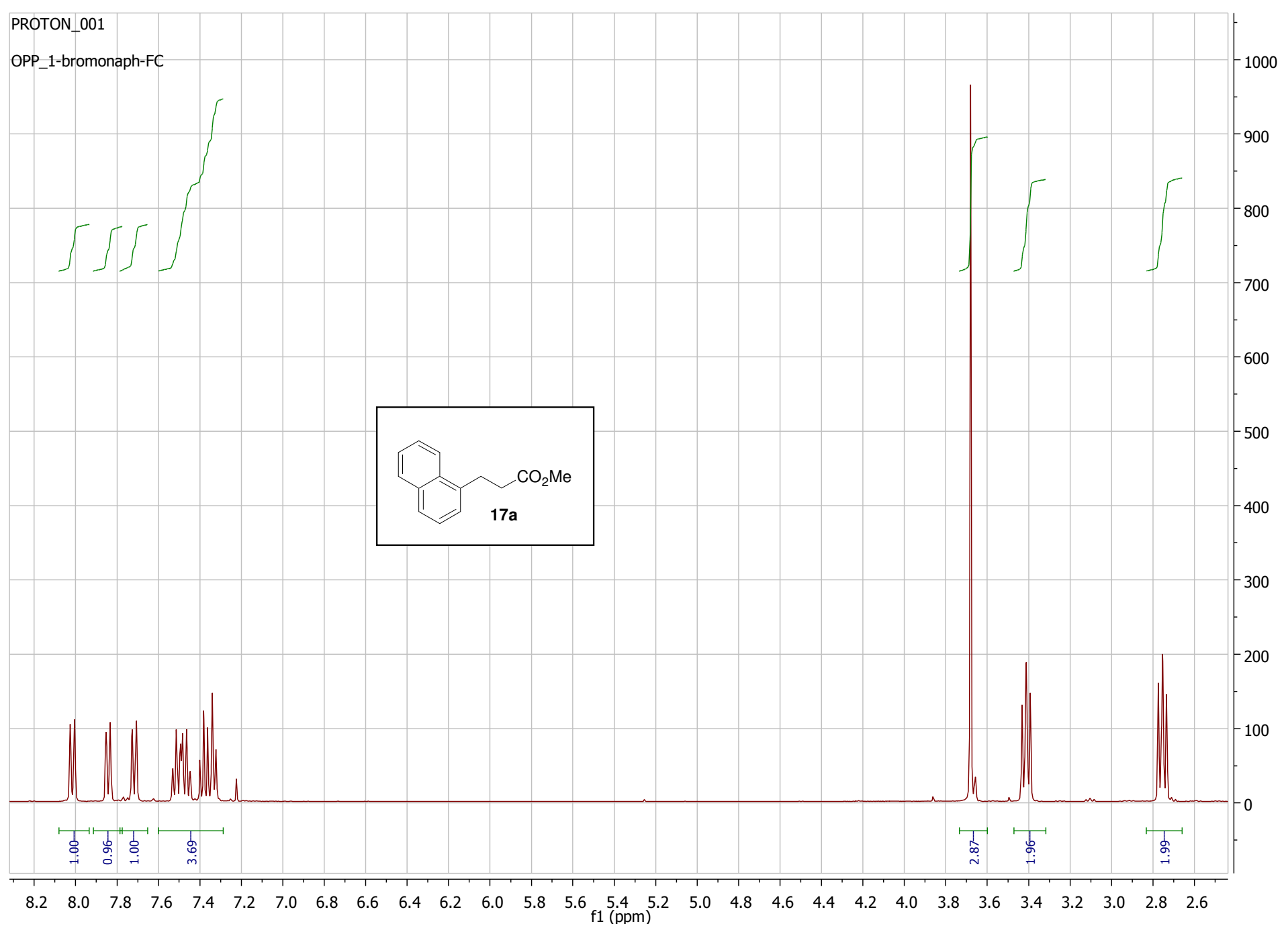


Methyl 3-(1-naphthyl)propanoate 17a (100 MHz, $\left.\mathrm{CDCl}_{3}\right)$

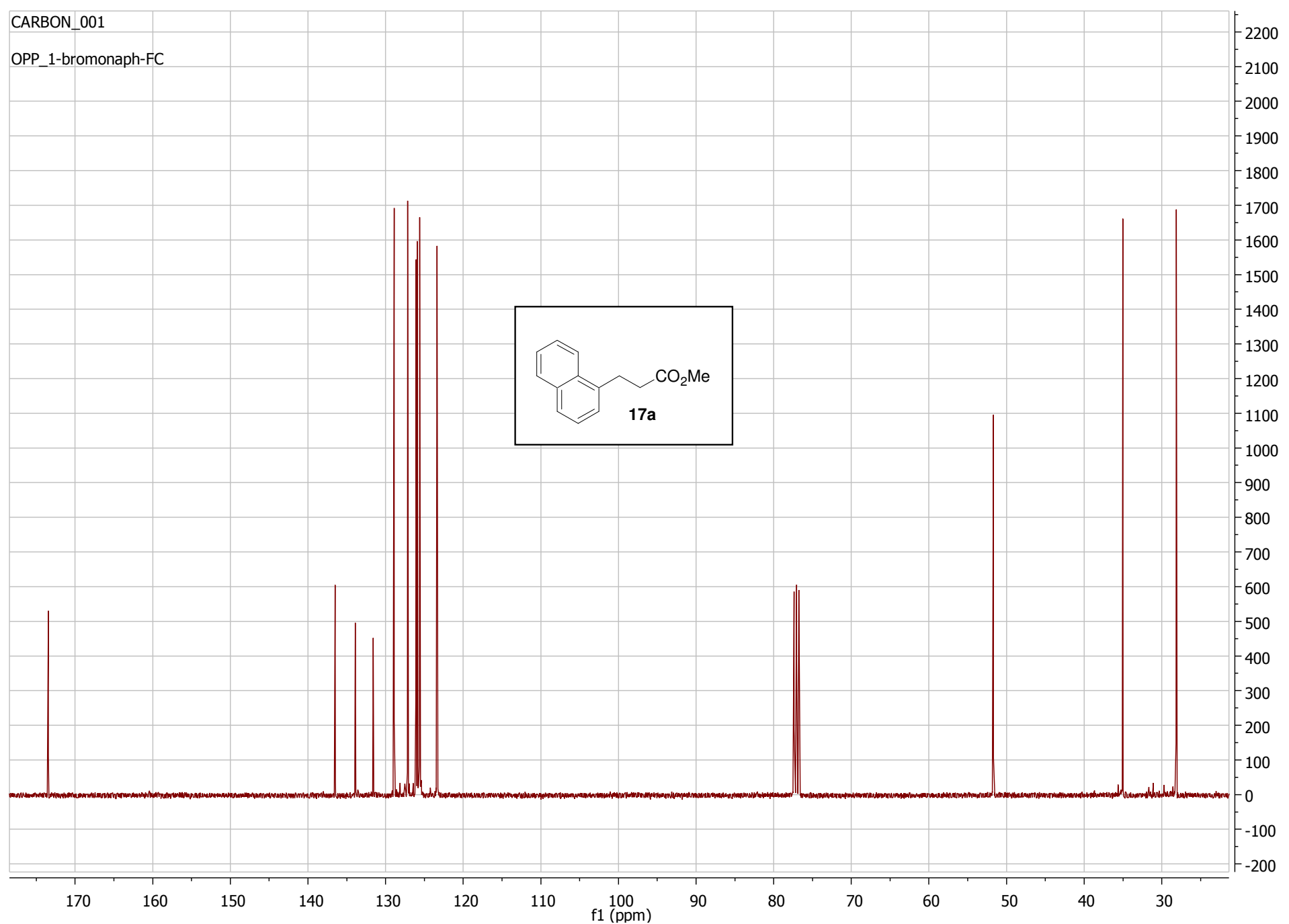


Methyl 5-(3,4-dimethoxyphenyl)pentanoate $\mathbf{1 7 f}\left(400 \mathrm{MHz}, \mathrm{CDCl}_{3}\right)$

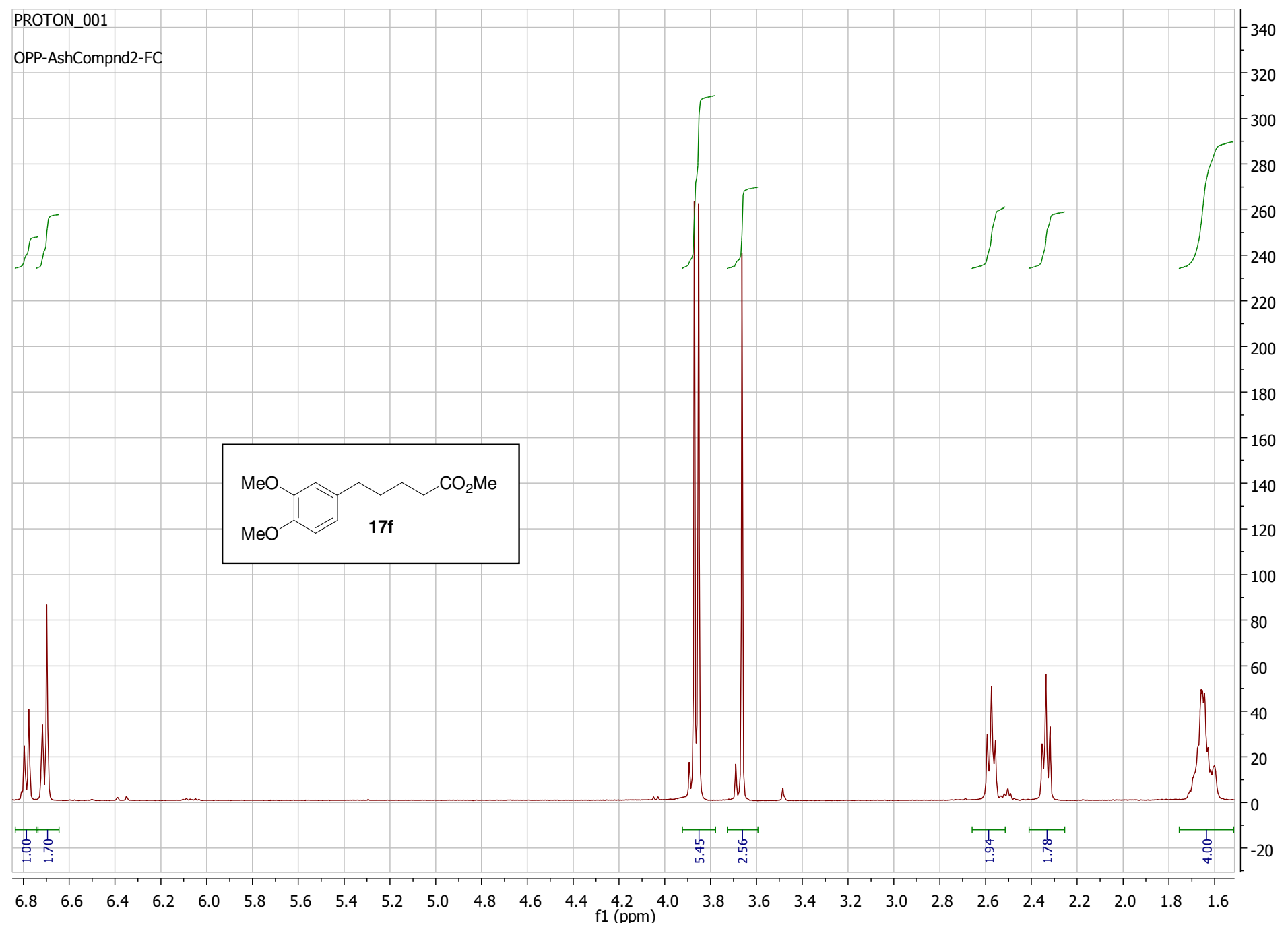


Methyl 5-(3,4-dimethoxyphenyl)pentanoate $17 \mathbf{f}\left(125 \mathrm{MHz}, \mathrm{CDCl}_{3}\right)$

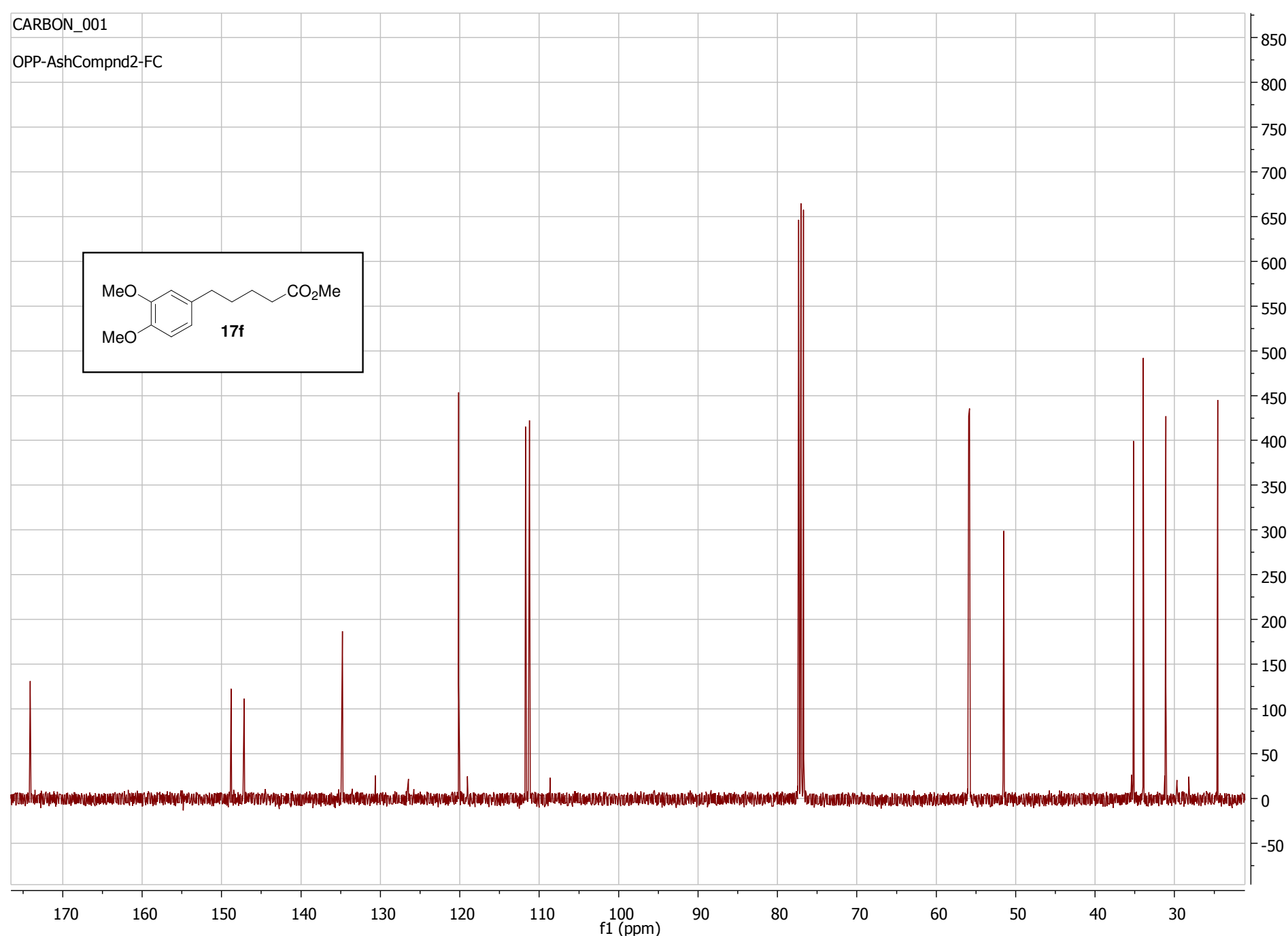


Methyl 3-(pyridin-3-yl)propanoate 17i (500 MHz, $\mathrm{CDCl}_{3}$ )

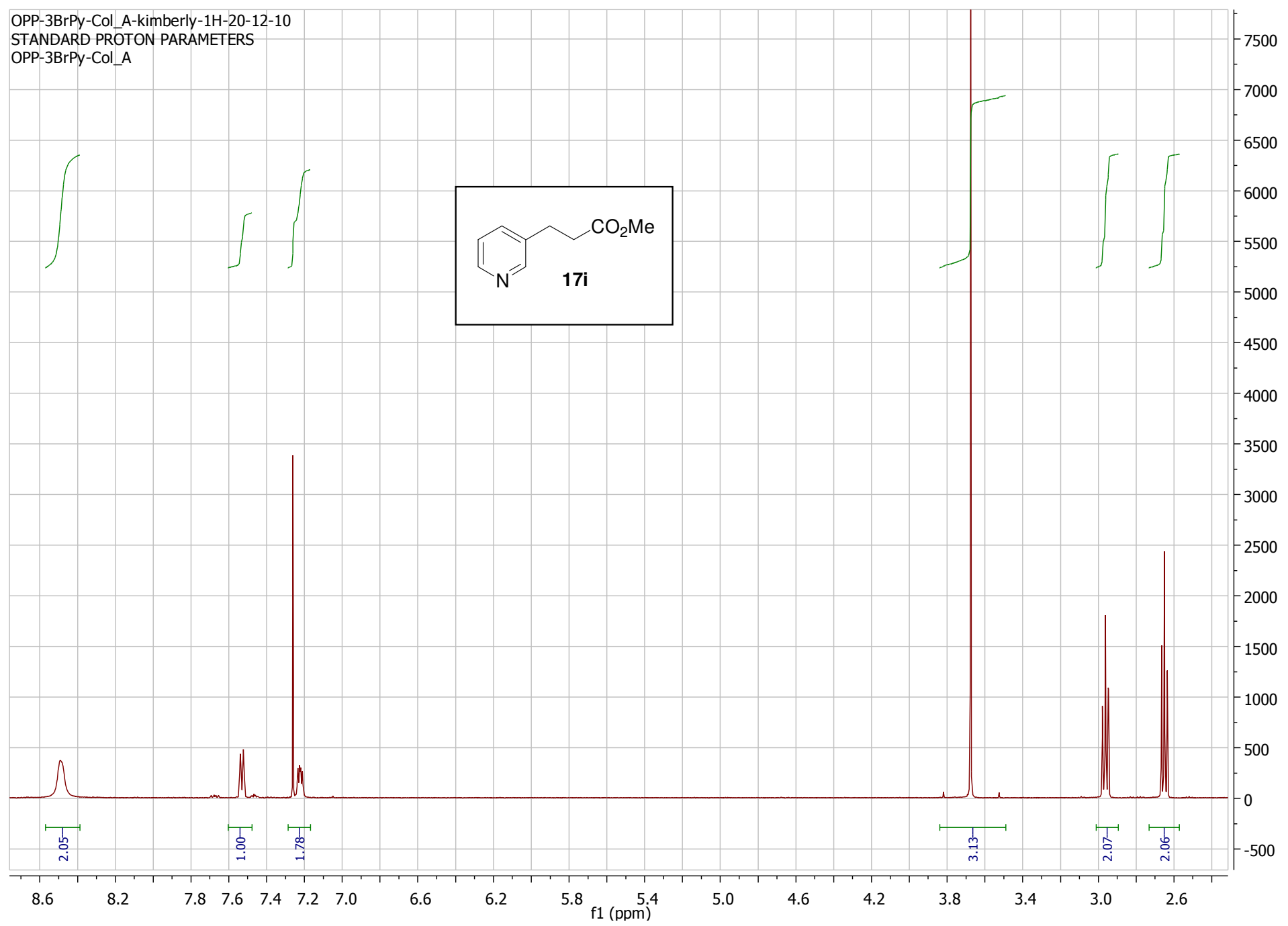


Methyl 3-(pyridin-3-yl)propanoate 17i (125 MHz, $\mathrm{CDCl}_{3}$ )

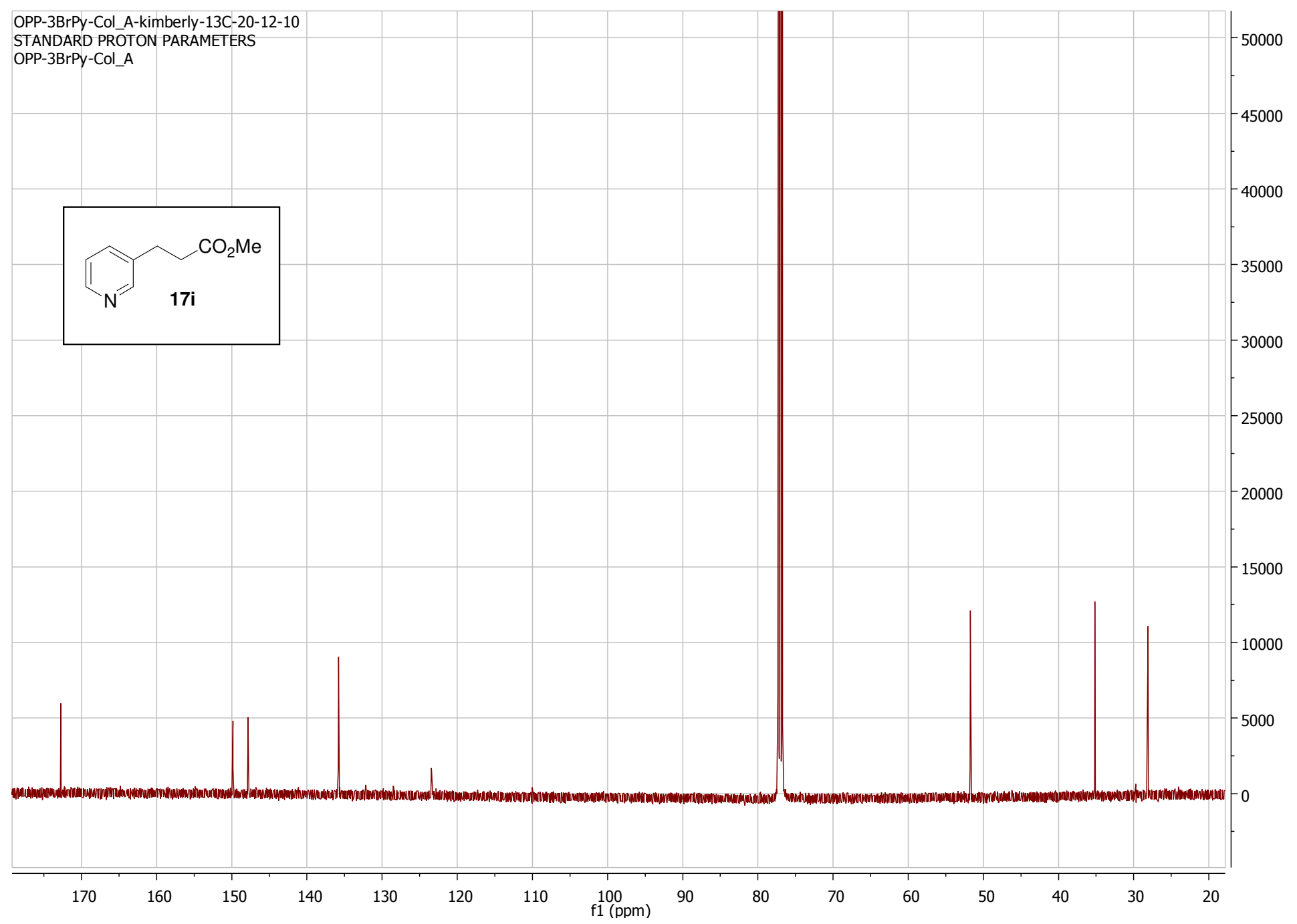


Methyl 3-(1,2,3,4-tetrahydroquinolin-3-yl)propanoate $\mathbf{1 7 j}$ (400 MHz, $\mathrm{CDCl}_{3}$ )

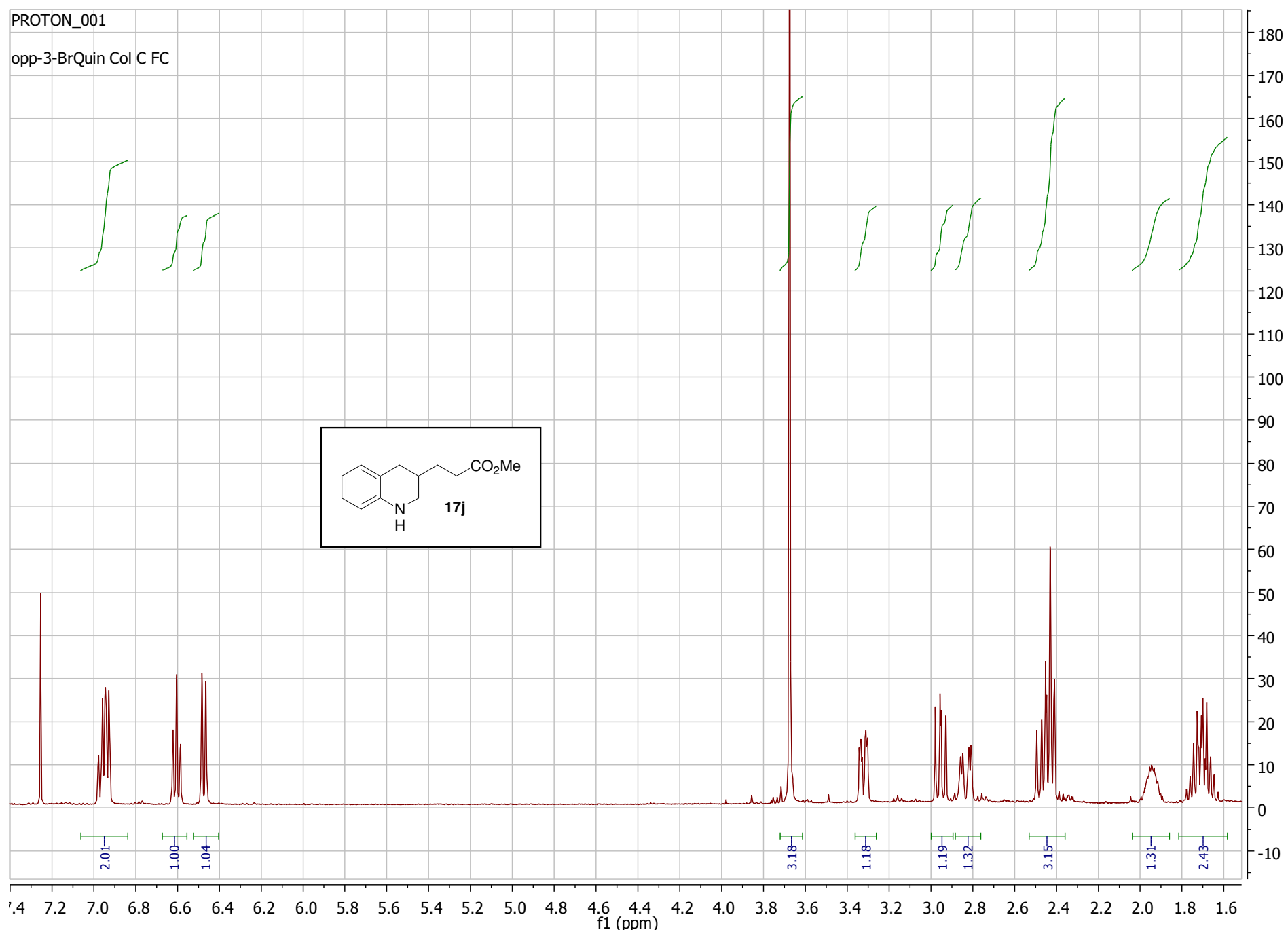


Methyl 3-(1,2,3,4-tetrahydroquinolin-3-yl)propanoate $\mathbf{1 7 j}\left(100 \mathrm{MHz}, \mathrm{CDCl}_{3}\right)$

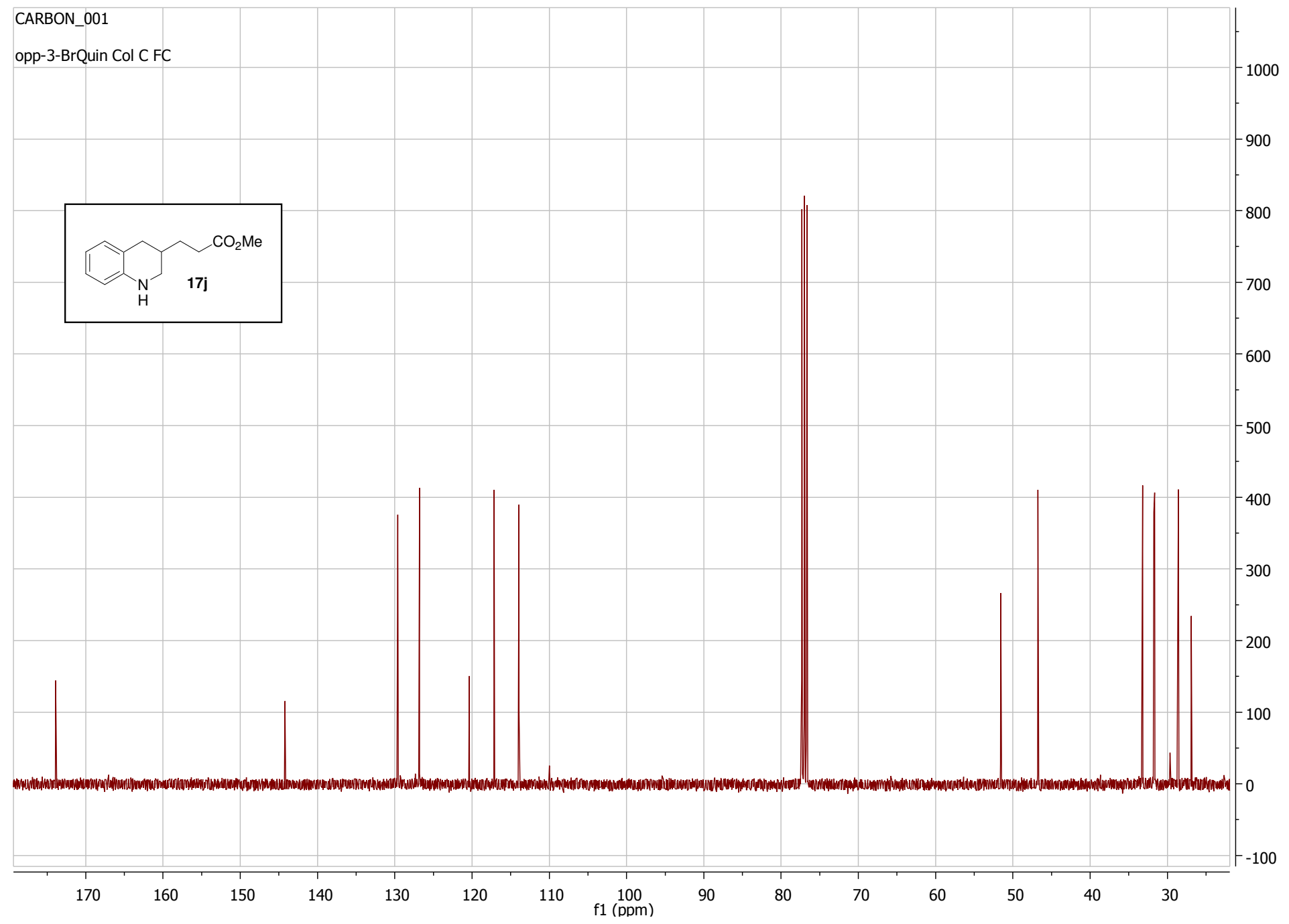


Methyl 3-(3-(trifluoromethyl)phenyl)propanoate 17k (400 MHz, $\mathrm{CDCl}_{3}$ )

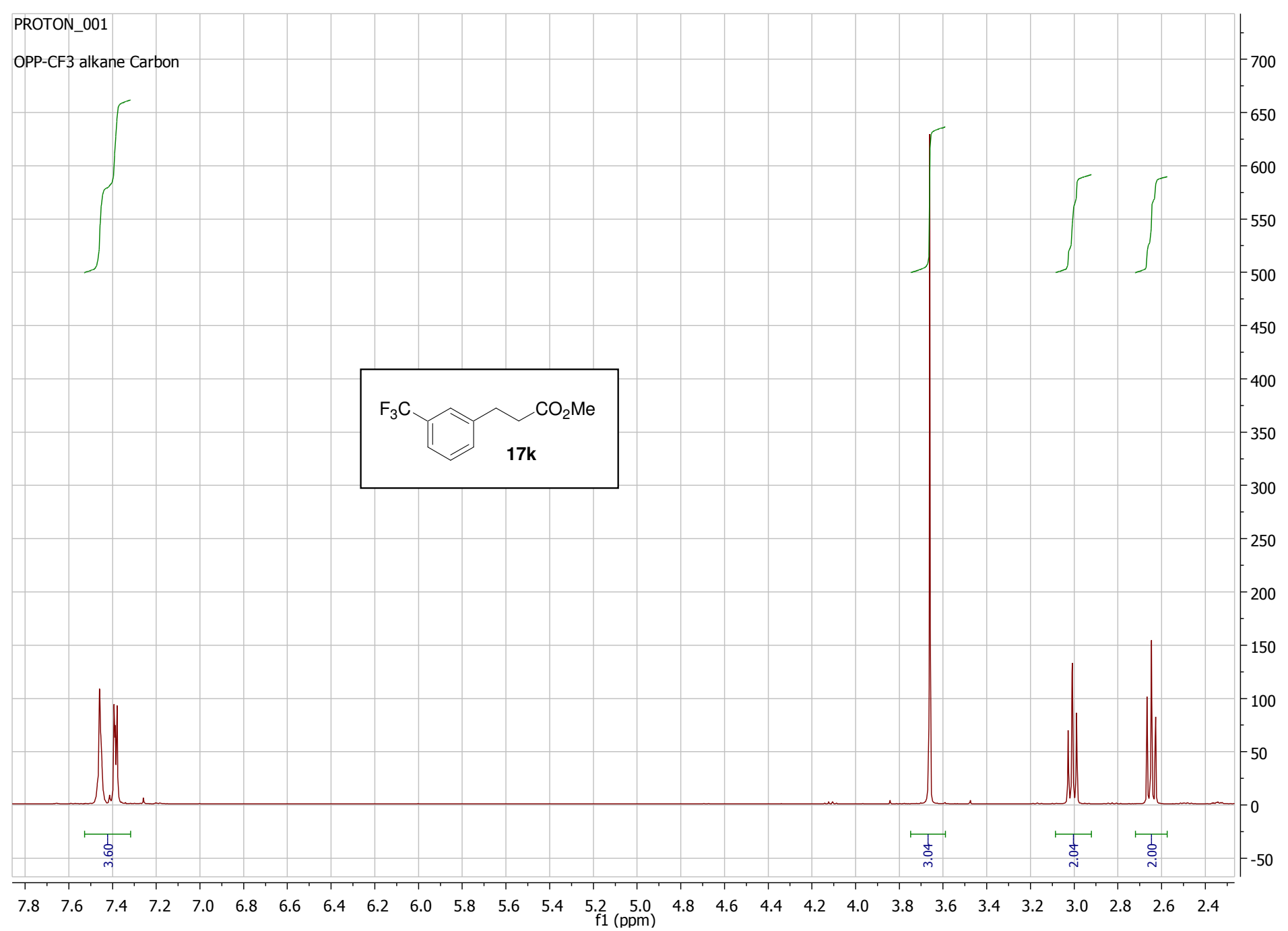


Methyl 3-(3-(trifluoromethyl)phenyl)propanoate 17k (100 MHz, $\mathrm{CDCl}_{3}$ )

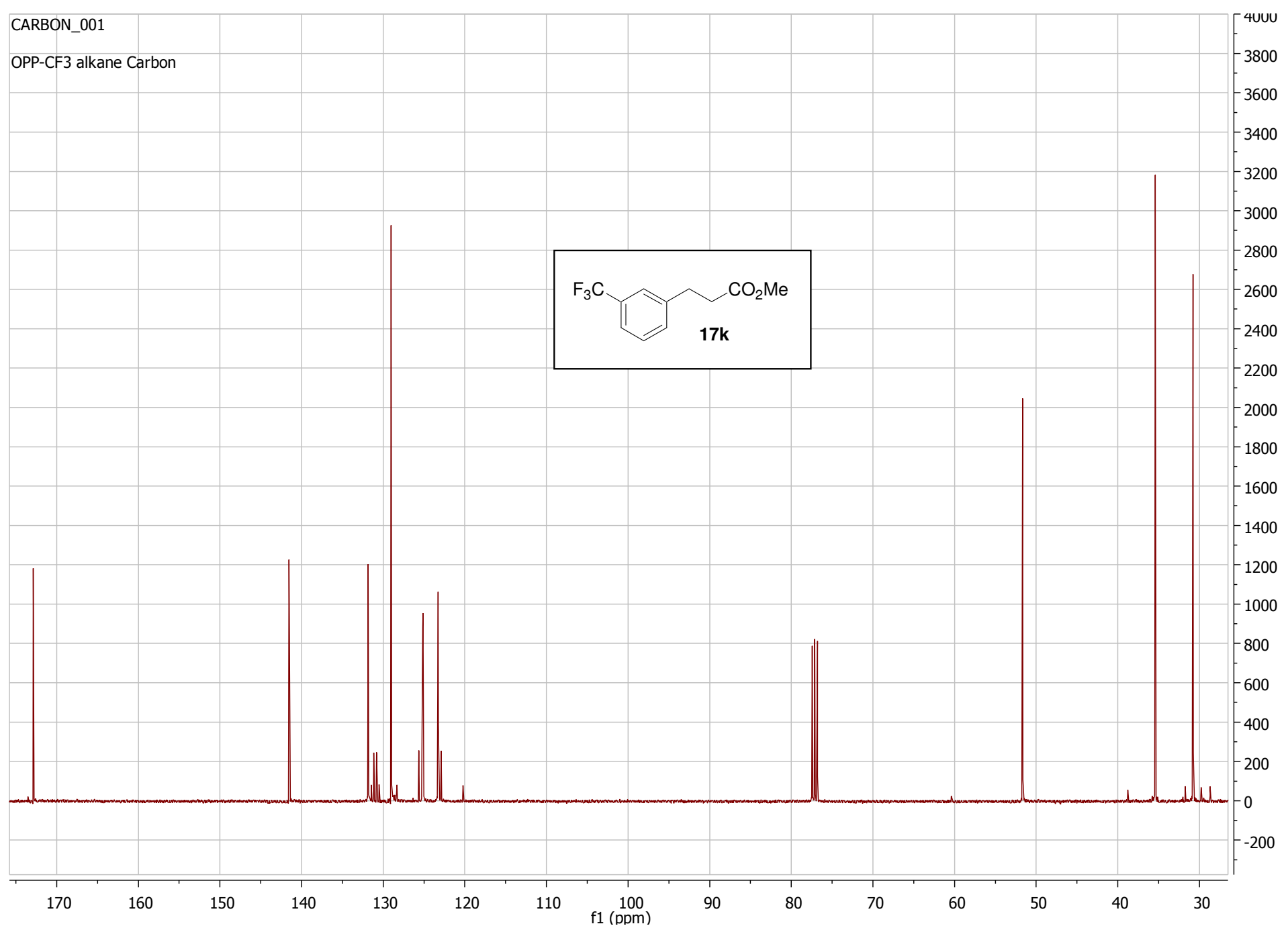


(R)- $N$-(1-(Naphthalen-1-yl)ethyl)-3-(3-(trifluoromethyl)phenyl)propanamide 18 (400 MHz, $\mathrm{CDCl}_{3}$ )

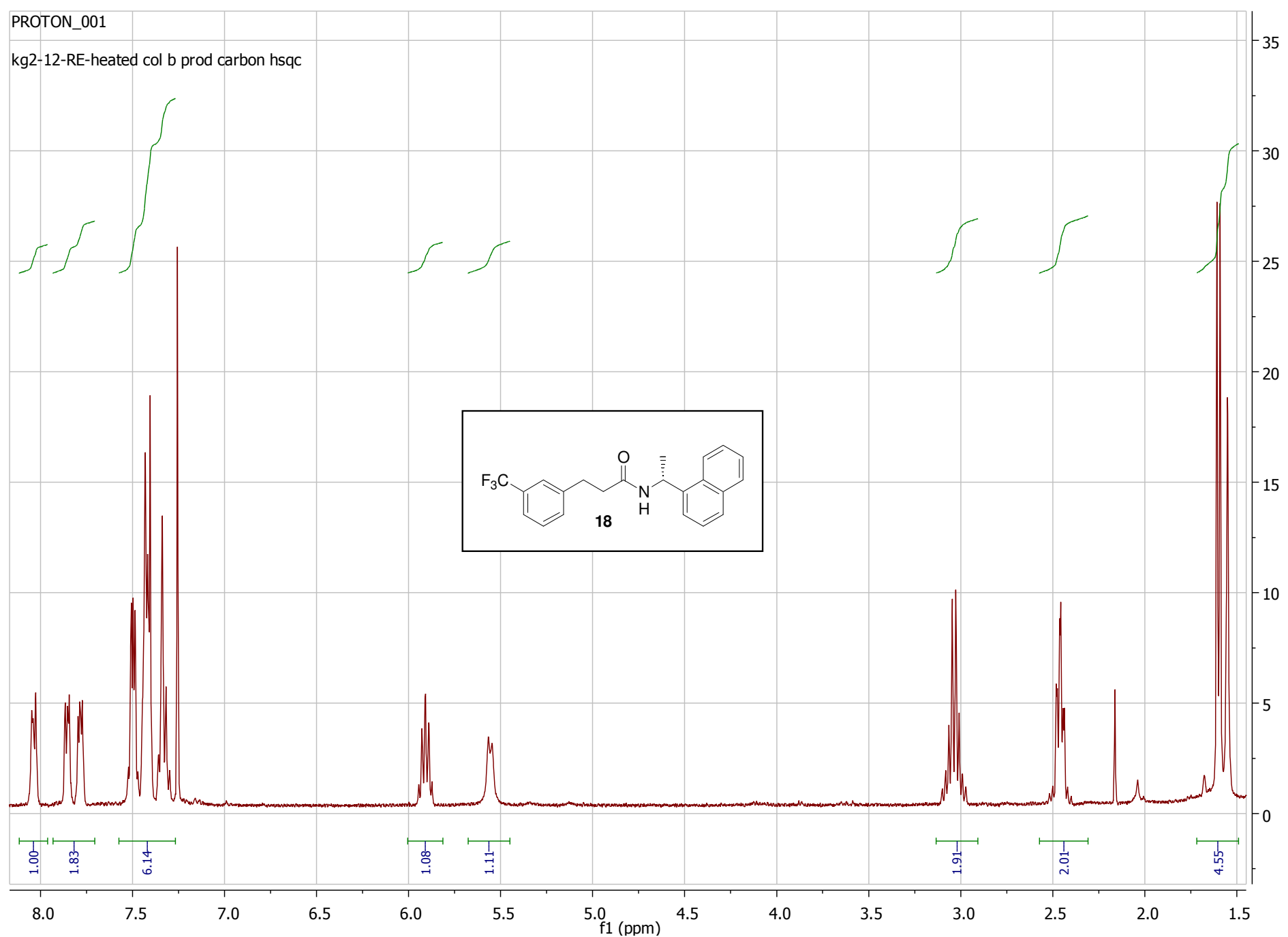


(R)-N-(1-(Naphthalen-1-yl)ethyl)-3-(3-(trifluoromethyl)phenyl)propanamide 18 (100 MHz, $\left.\mathrm{CDCl}_{3}\right)$

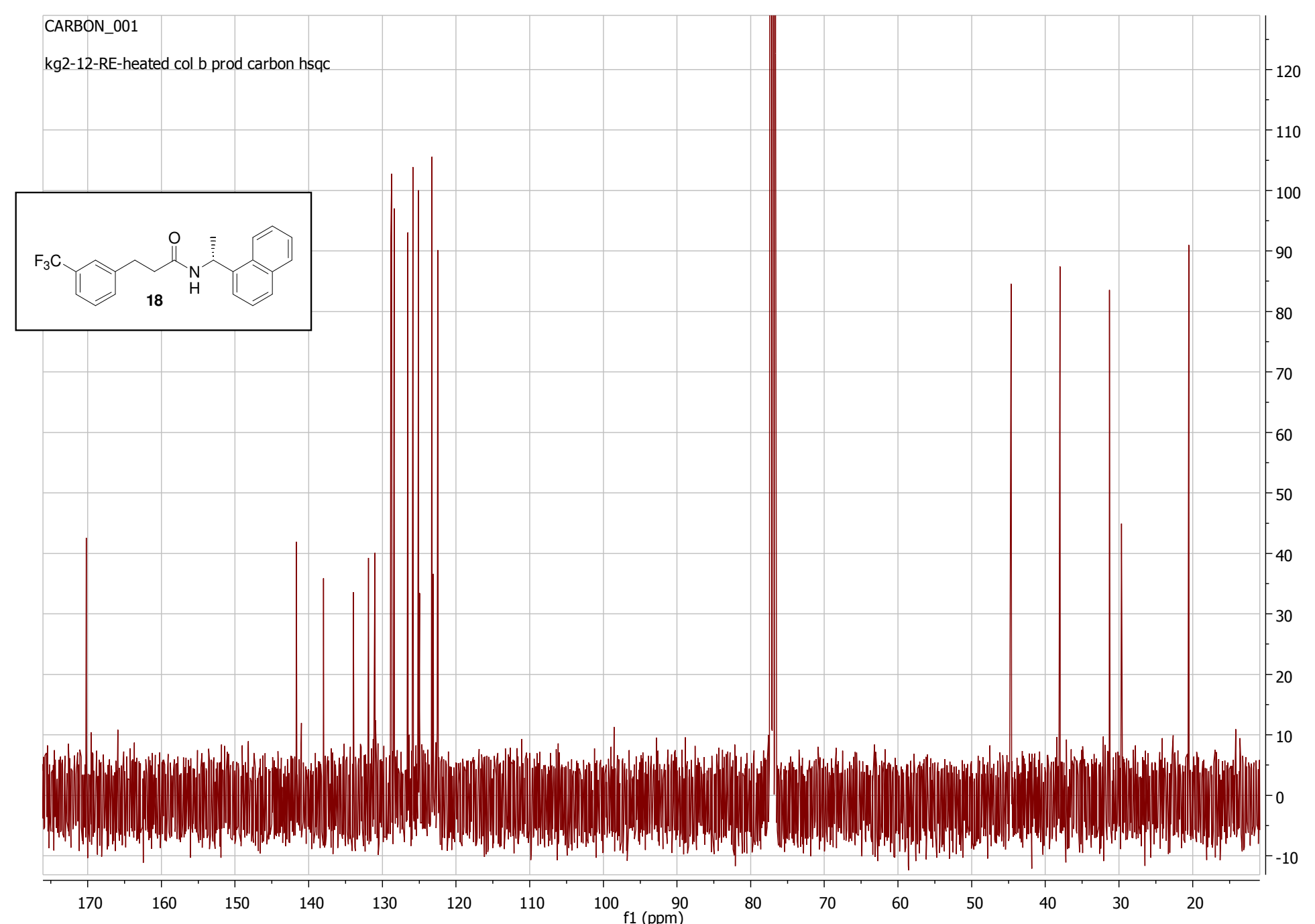


(R)-N-(1-(Naphthalen-1-yl)ethyl)-3-(3-(trifluoromethyl)phenyl)propan-1-amine (Sensipar) 19 (400 MHz, $\mathrm{CDCl}_{3}$ )

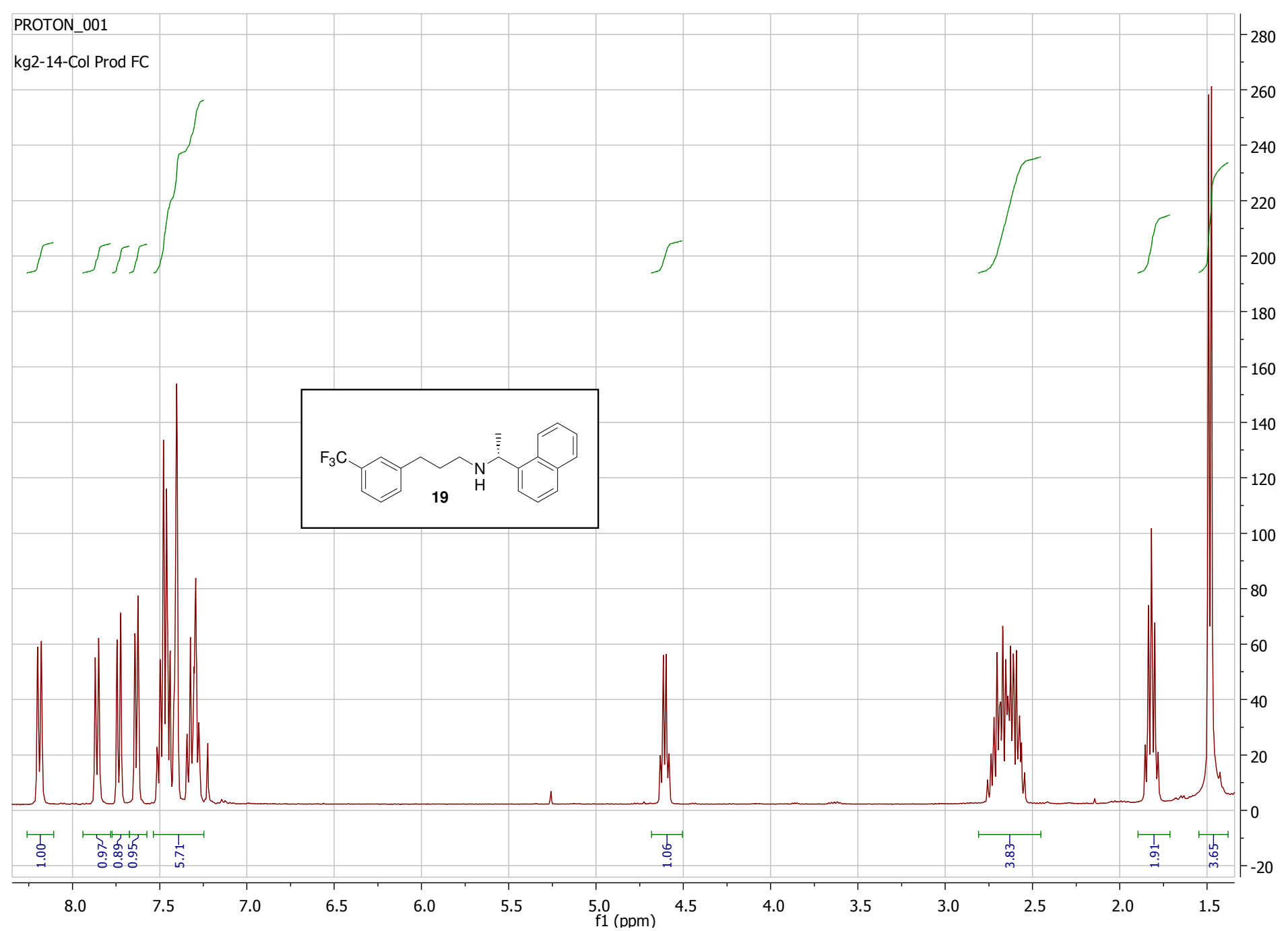


(R)- $N$-(1-(Naphthalen-1-yl)ethyl)-3-(3-(trifluoromethyl)phenyl)propan-1-amine (Sensipar) 19 (100 MHz, $\left.\mathrm{CDCl}_{3}\right)$

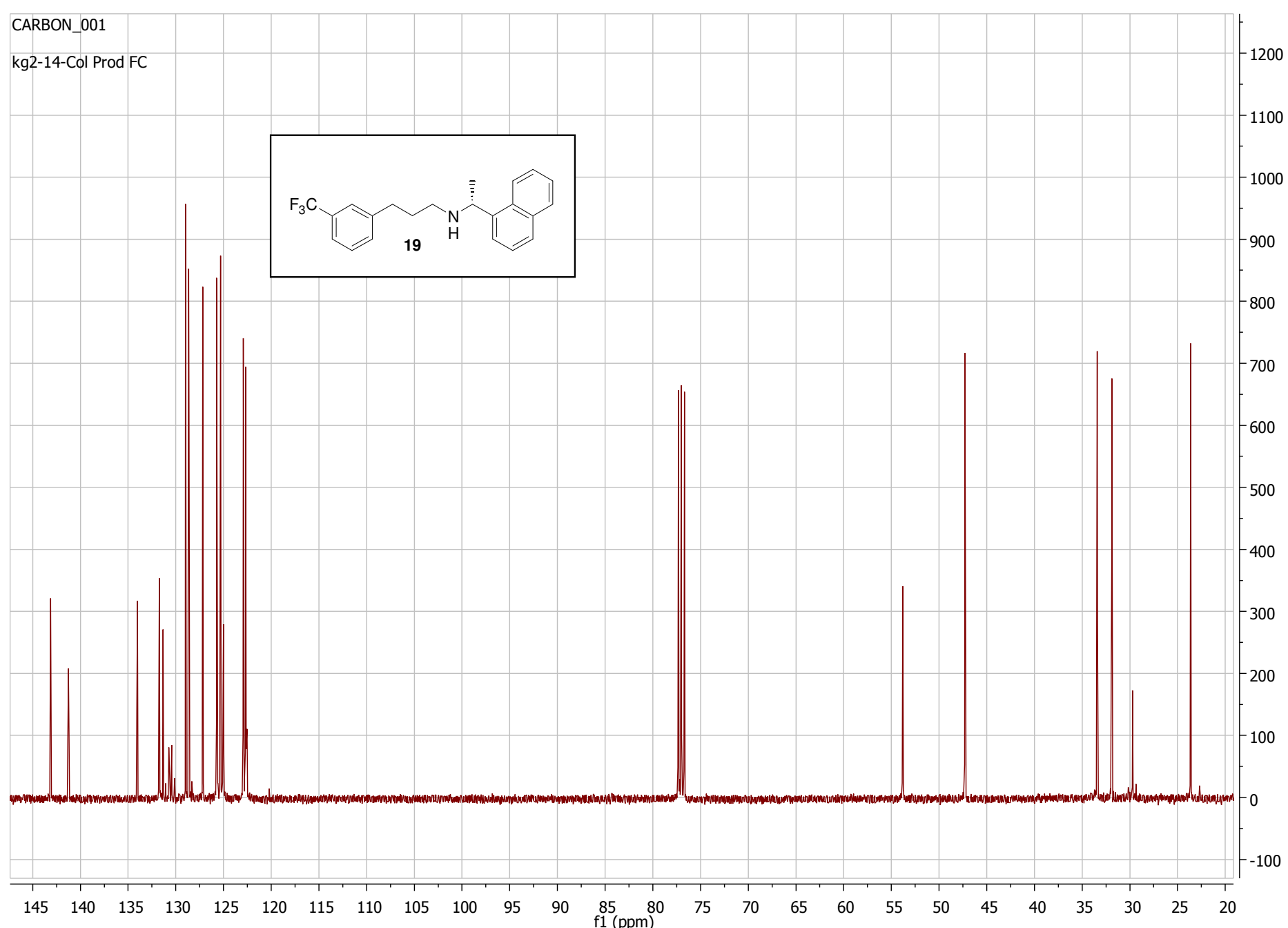


Mixture of methyl 3,3-diphenylacrylate 20 and 3-Phenylpropionic acid methyl ester $\mathbf{1 5 a}\left(400 \mathrm{MHz}, \mathrm{CDCl}_{3}\right)$

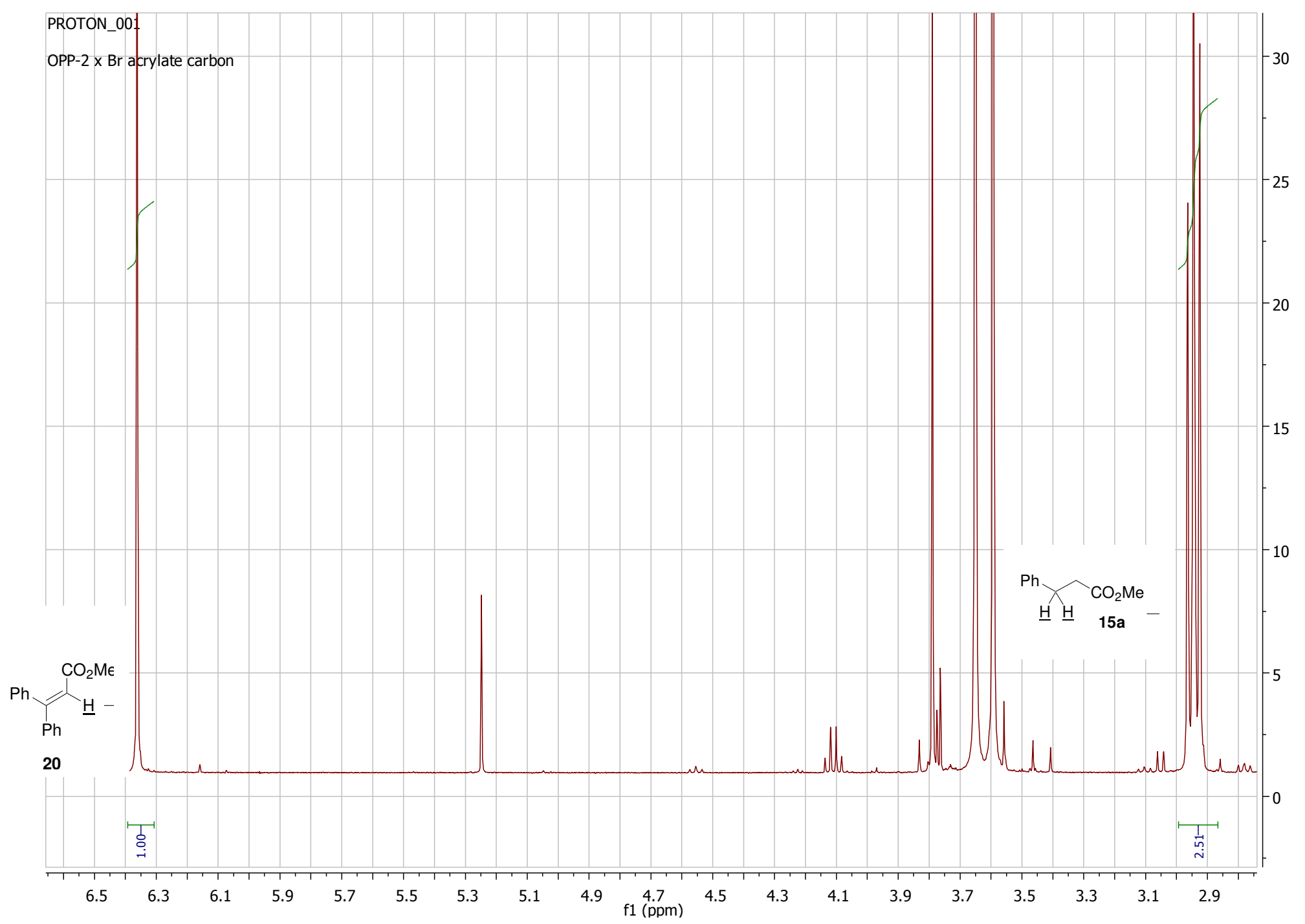

